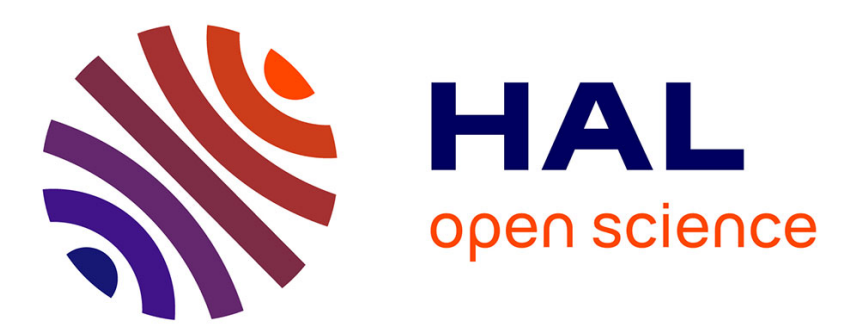

\title{
La vente des tombes à travers l'épigraphie de la Rome chrétienne (IIIe-VIIe siècles): le rôle des fossores, mansionarii, praepositi et prêtres \\ Jean Guyon
}

\section{- To cite this version: \\ Jean Guyon. La vente des tombes à travers l'épigraphie de la Rome chrétienne (IIIe-VIIe siècles): le rôle des fossores, mansionarii, praepositi et prêtres. Mélanges de l'Ecole française de Rome - Antiquité, 1974, 86 (1), pp.549-569. 10.3406/mefr.1974.972 . halshs-01465017}

\section{HAL Id: halshs-01465017 \\ https://shs.hal.science/halshs-01465017}

Submitted on 10 Feb 2017

HAL is a multi-disciplinary open access archive for the deposit and dissemination of scientific research documents, whether they are published or not. The documents may come from teaching and research institutions in France or abroad, or from public or private research centers.
L'archive ouverte pluridisciplinaire HAL, est destinée au dépôt et à la diffusion de documents scientifiques de niveau recherche, publiés ou non, émanant des établissements d'enseignement et de recherche français ou étrangers, des laboratoires publics ou privés. 


\section{La vente des tombes à travers l'épigraphie de la Rome chrétienne} (IIle, VIle siècles) : le rôle des fossores, mansionarii, praepositi et prêtres

Jean Guyon

\section{Résumé}

Jean Guyon, La vente des tombes à travers l'épigraphie de la Rome chrétienne (IIle-Vlle siècles): le rôle des fossores, mansionarii, praepositi et prêtres, p. 549-596.

Jusque vers 500, les ventes sont le fait des fossores, petits artisans indépendants, le plus souvent laïcs, mais assimilés aux clercs, qui assurent, à côté des inhumations, le développement, l'équipement et le gardiennage des catacombes: l'épigraphie tardive les nomme "résidants ", mansionarii.

Au Vle siècle, les tombes se concentrent autour des basiliques suburbaines gérées par des praepositi, dont certains sont clercs; la vente des tombes est de leur ressort.

A partir de 600, dans les églises urbaines où commencent les sépultures, les prêtres, qui jusque-là n'avaient joué qu'un rôle de surveillance, commencent à vendre des tombes.

\section{Citer ce document / Cite this document :}

Guyon Jean. La vente des tombes à travers l'épigraphie de la Rome chrétienne (IIle, VIle siècles) : le rôle des fossores, mansionarii, praepositi et prêtres. In: Mélanges de l'Ecole française de Rome. Antiquité, tome 86, n¹. 1974. pp. 549-596; doi : 10.3406/mefr.1974.972

http://www.persee.fr/doc/mefr_0223-5102_1974_num_86_1_972

Document généré le 09/05/2016 


\section{LA VENTE DES TOMBES À TRAVERS L'ÉPIGRAPHIE DE LA ROME CHRÉTIENNE (III -VII' SIĖCLES): LE RÔLE DES FOSSORES, MANSIONARII, PRAEPOSITI ET PRETTRES}

PAR

Jean Guron

Membre de l'Ecole

Sur les tombes chrétiennes, les dédicaces, comme les titres de propriété, ne manquent pas, et, sans atteindre à la précision de ceux des tituli paiens, les renseignements qu'ils fournissent sur les achats et les ventes permettent de dresser, au moins sommairement, un tableau du commerce des tombes dans les catacombes et les cimetières sub divo de la Rome chrétienne.

Le sujet a déjà été largement abordé par G.-B. de Rossi, dans sa Roma sotterranea qui demeure, après un siècle, la référence fondamentale ${ }^{1}$, mais les progrès des fouilles conduites par la Commission Pontificale d'Archéologie et publiées par la Rivista di Archeologia cristiana ${ }^{2}$, comme l'avancement de la publication de la Nova Series des Inscriptions christianae Urbis Romae, commencée par A. Silvagni et diligemment continuée par le Père A. Ferrua ${ }^{3}$, permettent aujourd'hui non seulement de dresser

1 G.-B. de Rossi, La Roma sotterranea cristiana descrita et illustrata (que je citerai en abrégé R.S.), 3 vol., Rome, 1864-1877, et notamment vol. III, p. $520 \mathrm{sq}$. pour les praepositi, mansionarii et fossores.

2 Rivista di Archeologia cristiana ( $R A C)$, éditée à Rome depuis 1924, qui a pris la suite du $N$ uovo Bulletino di Archeologia cristiana ( $N B A C$ ), Rome, 1895-1922, lui-même continuateur du Bulletino di Archeologia cristiana (BAC), Rome, 1863-1894, dirigé et animé par G.-B. de Rossi lui-même.

3 Les ICUR, NS, 5 vol. publiés à Rome, par A. Silvagni, I, Incertae originis, 1922; II, Viis Cornelia, Aurelia, Portuensi et Ostiensi, 1935, puis par 
un inventaire plus détaillé, mais aussi de présenter la question sous un jour différent, car on peut connaître désormais assez précisément la composition et l'histoire des groupes sociaux formés par les vendeurs de tombes.

Parmi les inscriptions indiquant les ventes, les seules inscriptions mentionnant le nom et la qualité $d u$ vendeur sont en effet en nombre suffisant pour fournir une image relativement précise. Il ne faut pas s'illusionner pourtant: dans l'univers des catacombes où une inscription sur huit seulement atteste une vente de tombe ${ }^{1}$, alors que la vente était sans doute la règle et l'inhumation gratuite l'exception réservée aux indigents, les inscriptions indiquant les noms des vendeurs sont tardives et restent fort rares: aussi est-ce un nombre infime de vendeurs dont le nom est conservé.

Pourtant, malgré cette sérieuse limitation due à la nature des documents utilisés, il est possible d'entrevoir clairement l'existence de trois grands groupes de vendeurs de tombes que l'on peut classer ainsi par importance numérique décroissante:

- les fossores dont le rôle dans la vente des tombes est attesté de $337 / 352$ (ICUR, NS, IV, 11751) à 465 (?) (ICUR, NS, I, 3240);

- les praepositi dont les interventions vont de 498 (ICUR, NS, II, 4998) à $561 / 566$ (?) (ICUR, NS, II, 5098);

- les prêtres, qui interviennent dans la vente des tombes dès 360/370 (ICUR, NS, III, 8441) et les vendent directement de 489 (BAC, 1882, p. 65) à 619 (R.S., III, p. 552).

Il convient d'ajouter à cette liste un achat à un mansionarius (ICUR, NS, I, 1987) dont je parlerai à l'occasion des fossores, quelques conces-

A. Silvagni et A. Ferrua, III, Via Ardeatina, 1956, enfin par A. Ferrua seul, IV, inter vias Appiam et Ardeatinam, 1964"et V, Via Appia, 1972, continuent la publication de G.-B. de Rossi, ICUR, I et II, Rome, 1857-61 et 1881, augmentée du supplément de J. Gatti, Rome, 1915.

Le présent travail repose sur cette documentation (augmentée de renseignements puisés essentiellement dans le $B A C$, le $N B A C$ et la $R A C$ pour les zones non encore publiées): ainsi s'expliquent les limites chronologiques retenues, les $I C U R$ s'arrêtant au début du VIIIe siècle.

$113 \%$ est en effet le nombre approximatif auquel m'a conduit le dépouillement des $I C U R$, en comptant à la fois les dédicaces (qui impliquent une intervention au moment de l'aménagement de la tombe) et les achats proprement dits. Je renonce à donner une estimation trop précise, car les restitutions ne permettent pas toujours d'être affirmatif: mieux vaut se contenter d'un ordre de grandeur assuré. 
sions de sépultures accordées par le Pape (sur lesquelles je reviendrai à propos des prêtres), et surtout quelques achats où la qualité des vendeurs n'est pas mentionnée: comme le formulaire et parfois la paléographie invitent à les ranger parmi les inscriptions des IVe et Ve siècles, je les étudierai avec les inscriptions des fossores.

Fossores et praepositi forment les deux groupes les plus intéressants, car la vente est pour eux une activité essentielle, ou habituelle, tandis qu'elle n'est qu'occasionnelle pour les prêtres. C'est donc sur eux surtout que portera cette étude, où j'essaierai d'examiner aussi bien leur rôle dans la vente des tombes que leur groupe social, et leur place dans l'église de Rome: c'est pourquoi, dans leur cas, j'ajouterai leurs épitaphes aux inscriptions indiquant les ventes ${ }^{1}$.

LES " FOssores" ET LEUR RÔLE DANS LA VENTE DES TOMBEs AUX IVe ET Ve SIÈCLES ${ }^{2}$

Employés à l'aménagement et à l'entretien des cimetières, et certainement aussi à l'inhumation, comme le montre une plaque du cimetière de Commodille, ICUR, NS, II, 6446, (Fig. 1) datée par le Père Bagatti de la seconde moitié du IVe siècle, leur rôle a été déterminant dès les origines des catacombes, qui sont leur œuvre.

Dans le courant du IIIe siècle, dans la catacombe de Callixte, on les voit figurer aussi bien sur une plaque (ICUR, NS, IV, 12228) que sur les peintures d'une des "chapelles des sacrements" ${ }^{3}$, mais leur apparition dans l'épigraphie est plus tardive et commence par des inscriptions funéraires. Sans doute ne faut-il pas les placer trop haut dans le temps,

1 Je groupe le plus intéressant pour la présente étude est celui des fossores, qui, à la différence des praepositi, se consacrent exclusivement à l'aménagement et à la vente des tombes: on a donc veillé à en dresser la liste la plus complète possible, et on leur a consacré les plus longs développements.

2 Je tiens à remercier de leur aide et de leurs conseils MM. les Professeurs Pasquale Testini, de l'Université de Rome et de l'Institut Pontifical d'Archéologie, qui a bien voulu me communiquer les résultats d'un premier dépouillement effectué par l'un de ses étudiants, et Charles Piétri, de l'Université de Paris-X, que ses recherches sur la Roma christiana ont conduit à étudier le rôle des fossores dans l'église romaine des premiers siècles: ses suggestions m'ont permis d'améliorer le présent travail qui, entrepris indépendam. ment, m'a conduit à des résultats très voisins du sien.

3 G.-B. de Rossi, R.S., Tavole, II, pl. XVII. 
Fig. 1 - Cimetière de Commodille. Plaque funéraire représentaxt Un "Fossor" avec ses instruments de travall, "Piccone" et iampe a huILe. A ses pieds, un CORPS daNs son IINCEUl.

car on sait que le formulaire très simple des plus anciennes épitaphes chrétiennes ne mentionne que rarement le métier du défunt; sans qu'on puisse toujours les dater avec précision, les épitaphes n'indiquant que le nom et la qualité de fossor sont pourtant antérieures à celles qui mentionnent leurs interventions dans les ventes de tombes, et commencent dès la mort de Constantin (la première vente très exactement datée est de 338): aussi la date de 337 est-elle une césure tout indiquée pour un classement chronologique sommaire des inscriptions de fossores.

Catalogue topo-chronologique des inscriptions de fossores ${ }^{1}$.

Via Aurelia

Saint-Pancrace

1. avant 337: FELIX, fossarius, épitaphe ICUR, NS, II, 4333.

2. après 337: BICTORINUS et EXUPFRUS, ICUR, NS, II, 4344. son collègue (vente)

1 Ce catalogue comprend les inscriptions des fossores (épitaphes et ventes) et celles des ventes effectuées par des vendeurs dont la qualité n'est pas précisée, mais que le formulaire invite à placer aux IVe ou Ve siècle. 
Via Portuensis

Pontien

3. $a^{0} 430$ :

EPOLYTUS, fossor (vente)

$I C U R, N S$, II, 4514.

Via Ostrensis

Area Lucinae

Après 337:

4. $\mathrm{a}^{\mathrm{o}}$ 432? 484? 487? : REDEM[PTCS] (vente) ICUR, NS, II, 5865.

5. pour 6 sous ${ }^{1}$ )

AD[EO]DATUS, fossor (vente) ICUR, NS, II, 5855.

Commodille

Après 337:

6. $\mathrm{a}^{\circ}$ 380: $\left.\mathrm{LA}\right] \mathrm{LR}=$ Laurus?, fossor (vente) ICUR, NS, II, 6033.

7. a 398: PREIECTICIUS, fossor (vente) ICUR, NS, III, 8647.

8. vers 398: Le même (PROIECTICILS) ICUR, NS, III, 8655.

$\left.\begin{array}{ll|l}\text { 9. } \mathrm{a}^{\circ} \text { 426: } & \begin{array}{l}\text { BURDO } \\ \text { MICINLS } \\ \text { MUSCORUTIO }\end{array}\end{array}\right\} \begin{aligned} & \text { fossores } \\ & \text { (vente pour } \\ & 1 \text { sou et demi) }\end{aligned}$

10. vers 426: I,e même MUSCL[R]UTIO

11. ADEODATUS, fossor (vente) (vente) $=6106-6107$.

12. en présence d'un prêtre)

ICUR, NS, II, 6077 .

13.

14.

AN]DREAS? (vente?) ${ }^{2} \quad I C U R, N S$, II, 6103.

15.

EXU]PERA NTIUS, fossor (vente) ICUR, NS, II, 6104.

MULIA, (vente) ICUR, NS, II, 6105.

PREIICTUS $=$ Proiectus? (vente) $^{3}$ ICUR, NS, II, 6267.

Il suit l'ordre du recueil des $I C U R, N S$, les inscriptions d'origine incertaine étant toutefois rejetées à la fin. Pour chaque cimetière, j'ai séparé les inscriptions en distinguant celles qui se placent avant 337 et celles qui se placent après. Dans chaque série, on trouvera en tête les inscriptions datées.

Il va de soi que tout classement chronologique comporte une part d'arbitraire: je me suis appuyé sur le formulaire et à l'occasion sur la paléographie pour l'établir.

D'autre part, la localisation des inscriptions n'est pas toujours certaine lorsqu'elles sont connues seulement par des copies anciennes dont les indica. tions sont parfois contradictoires. Ailleurs, j'ai suivi les indications des éditeurs des $I C V R$.

1 L'éditeur restitue praepositus sur cette inscriptions lacunaire. Les datations qu'il propose me font préférer la restitution fossor.

${ }^{2}$ Le nom, comme la qualité de fossor? sont des restitutions hardies de l'éditeur pour une inscription très mutilée: peut-être faut-il rejeter ce fragment? 3 On pourrait également proposer Preiecticius et l'identifier avec le no 7 . 


\begin{tabular}{|c|c|c|}
\hline 16. & $\begin{array}{l}\text { SEMPAO[NILS }]=\text { Sempronius? } \\
\text { (vente) }\end{array}$ & ICUR, NS, \\
\hline & VICTORINLS (vente) & $\begin{array}{l}\text { ICUR, NS, } \\
\text { ICUR, NS, }\end{array}$ \\
\hline & $\begin{array}{l}\text { fossor (eosur) dont le nom manque } \\
\text { (vente) }\end{array}$ & \\
\hline & omnis fossores, témoins à une vente & ICUR, \\
\hline
\end{tabular}

Via Ardeatina

Domitille

20. avant 337: ILNILS | fossores, sur une ICLR, NS, III, 9143. Après 337:

21.

22.

23.

24.

25.

26. SERGIUS $\}$ même épitaphe ${ }^{1}$

ALR. CONSTANTINUS ) (vente) ICUR, NS, III, 8202. ALR. BIATURINLS DIOGENES, fossor, épitaphe et ICUR, NS, III, 6449. peinture (milieu IVe siècle?)

I.ALR[C'S] (vente)

ICUR, NS, III, 7677d. ICUR, NS, III, 7760. ICUR, NS, III, 8481 . ICUR, NS, III, 8485.

ENtre Via Appia et Via ardeatina

Callirte

27. avant 337: ILNILS, fossor Aventinus (épitaphe) $^{2}$

ICUR, NS, II, 4629.

$\Lambda$ près 337 :

28. a 378: MARTINIANES, fossor (vente) ICUR, NS, IV, 11722.

29. a 394 ? 396? 402?: AS'TA[ERIIS?] (vente) ICUR, NS', IV, 9580.

30. a 402/407?: fossor, dans le carmen ICUR, NS, IV, 9268.

31 . d'Innocentius ${ }^{3}$

ICONIUS, fossor, sans doute, signe, ICUR, NS, IV, 9542. plusieurs graffiti.

(Le premier avec chrisme)

ICUR, NS, IV, $9534 \mathrm{a}$.

ICUR, NS, IV, 9920 .

1 La localisation précise de cette inscription, connue seulement par une copie de Boldetti est incertaine; alle appartient sûrement en tout cas à la zone de l'Appia ardéatine.

2 Boldetti donne deux provenances: Pontien, p. 65 et Gallixte, p. 62, et l'on sait que cette dernière localisation elle-même est floue, la toponymie de cette zone ayant beaucoup varié au cours des âges.

3 Marini donne également Cyriaque comme localisation. D'autre part, la datation que j'indique est celle proposée de façon assez aventureuse par E. Diehl, Inscriptiones Latinae Christianae Veteres (ILCV), 3 vol., Berlin, 19241931. Pas plus que la date, le sens du texte n'est clair: le défunt, Innocentius, et le fossor du v. 9 sont-ils une seule et même personne? 
32.

33.

34.
QUINTLS, fossor (vente) fossor, dans un texte très mutilé fossor? (vente pour 4 sous)
ICUR, NS, IV, 9441.

$I C U R, N S, I V, 11523$.

$I C U R, N S, I V, 11231$.

Marc et Marcellin

Avant 337 ou autour de cette date:

35. $a^{\circ} 337 / 352$ : [ALEXAN]DER, fossor (vente sub ICVR, NS, IV, 11751. Iulio a[ntistite $]$ )

36. vers 337/52: Le même ALEXANDER (vente) ICUR, NS, IV, 11991. 37. C. PICE[NTILS] FORMO[NSLS] ICUR, NS, IV, 11973. fossor, épitaphe

Après 337:

38.

39.

40.
A]PER (vente)

GENIALIS (vente)

I.AURENTIUS (vente)
ICUR, NS, IV, 11941.

ICUR, NS, IV, 11985.

ICUR, NS, IV, 11960.

Cimetière près de l'Ardéatine

Après 337:

41.

42.

RUTICLLUS (vente)

$I C U R, N S, I \mathrm{~V}, 12283$.

43.

I se même (RUSTIC[..) (vente)

ICUR, NS, IV, 12336.

$I C U R, N S, I V, 12339$.

Près de la basilique du Pape Marc

44. après 337: FELIX, fossor (vente)

ICUR, NS, IV, 12458.

Inscriptions de la surface et d'origine imprécise

Après 337:

45. a 403: URSUS, fossor (vente)

46. a 513?: PETRUS? fossor, épitaphe ${ }^{1}$

$I C U R, N S, I V, 12425$.

ICUR, NS, IV, 12527.

Via Apria

Saint-Sébastien

Après 337:

47. a 373: QUOJDVLLDELS, fossor (vente) ICUR, NS, V, 13107. 48. FLORENTIUS, fossor (vente) ICUR, NS, V, 13198. 49. $\langle\mathrm{Q}\rangle \mathrm{O}\langle\mathrm{DR}\rangle \mathrm{ATCS}$ ?, fossor (vente) ${ }^{2} I C U R, N S, \mathrm{~V}, 13150$.

1 Le nom exact du défunt, comme la date proposée, sont également conjecturaux.

2 O. Marrucchi, NBAC, 1912, p. 140 lisait Ocapatostanees. L'éditeur des $I C U R$ coupe le nom en deux, et, s'il garde Stanees comme un second nom propre, propose pour le premier la restitution que j'indique: il est clair qu'elle reste conjecturale. 
Saint-Sébastien (surface)

50. avant 337?: LEO, fosor, épitaphe pour lui et ICUR, NS, V, 13568. sa virginia

51. après 389: $\mathrm{E} \operatorname{CSE}[\ldots$

\begin{tabular}{l|l} 
FORTUNIO & $\begin{array}{l}\text { vriers travaillant à } \\
\text { MAXIMLS } \\
\text { MCSICLS }\end{array}$ \\
LRSLS & $\begin{array}{l}\text { la Platonia sous la } \\
\text { direction de } \\
\text { Musicus }\end{array}$
\end{tabular}

52. sans date précise: fo]ssor, fragment

ICUR, NS, V, $13735 \mathrm{~g}$.

Prétextat

Après 337:

53. a 400 ?:

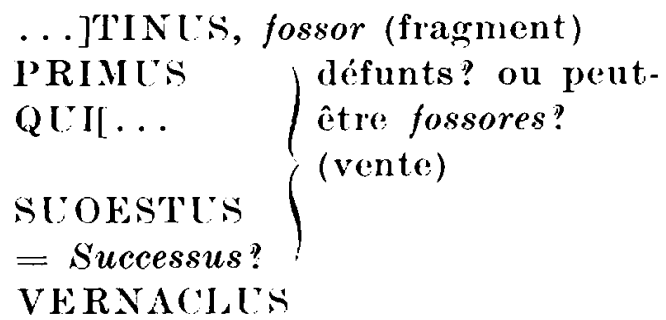

Via ILATINA

Gordien

Après 337:

55. $a^{0} 400$ :

CALEVILS (vente)

ICUR, I, 489.

56.

SICL: (vente)

Muratori ${ }^{1}$, 1947,3.

Apronianus

57. après 337: fosor anonyme (vente)

R.LC, 1939, p. 224.

Via Labicana

P'ierre et Marcellin

58. date indéterminée: [V]ICTORINLS, fossor (acclamation)

NBAC, 1901, p. 168.

59. après 337: ARIONTATME, fossor (vente) ${ }^{2}$ Muratori, 1930, 8.

Via 'Tiburtina

\section{Cyriaque ou Saint-Laurent}

Avant 337:

60.

FESTL's, epitaphe avec représen- Boldetti ${ }^{3}$, p. 369. tation du fossor assis

1 I.-A. Muratori, Novus Thesaurus Veterum Inscriptionum, 4 vol., Milan, $1739-1742$.

2 Je nom est très incertain: Marangoni lisait ann $p l, m$. $l x$ à la place de la lecture de Muratori.

3 A. Boldetti, Osservazioni sopra $i$ cimiteri de santi martiri ed antichi cristiani di Roma, t. I, Rome, 1720. 
61.

62.

$$
\begin{aligned}
& \text { ERCLIIIS ？ fossores } \\
& \text { TIGRINIANUS? (ंpitaphe) } \\
& \text { PETRLS, fossor, épitaphe }
\end{aligned}
$$

Après 337:

63. $a^{0} 400$ :

64. $a^{0} 403$ :

65. $a^{\circ}$ 405:

66.

67.

68.

69.

70.

71.

72.

73.

74.

75. 75 bis

CEILRINLS, fossor (vente)

CIAADDICS (vente)

..]V[.. fossor au nom rasuré (vente)

ANASTASIUS (ou ATHANASIl's)

ANTHIOCHIUS

APRO
BIATOR (vente)

DELFINUS (vente pour 2 sous)

HYIARINUS (vente)

fossores

Le même (HILA[...), dans une

vente (au même client?) ${ }^{2}$

ILE, fossor (vente)

MONTANUS (vente)

SAFARGICS, fossor (vente)

fossor, (vente), sur un fragment

fossor (vente) (texte mutilé, mais

le nom est absent)

Cyriaque ou Hippolyte

\section{SUSTUS}

(vente) ${ }^{1}$

Via Nomentana

Sainte-Agnès

76. Au temps de Constantin?: DEBESTLS

(épitaphe avec chrisme)

Cimetière Maius

Avant 337?:

77.

$$
\left.\begin{array}{l}
\text { CALLIGONUS } \\
\text { MAIUS } \\
\text { PROCILS }
\end{array}\right\} \begin{aligned}
& \text { épitaphe d'une Marucchi }{ }^{4}, \text { p. } 407 . \\
& \text { famille de } \\
& \text { fossores }^{5}
\end{aligned}
$$

1 Les leçons divergent sur le premier nom: Bosio donne Anastasius, et Fabretti Athanasius (Diehl, loc. cit.).

${ }^{2}$ G. Bovini, Sant'Ippolito, dottore e martire del terzo sec., Rome, 1943, p. 193, no 46, attribue cette inscription au cimetière d'Hippolyte. Peut-être faudrait-il également y localiser l'inscription précédente.

${ }^{3} \mathrm{G}$. Marchi, Monumenti delle arti cristiane primitive, Rome, 1844, p. 85, hésite sur la localisation précise.

4 O. Marucchi, Le catacombe romane (œuvre posthume éditée par F. Iosi), Rome, 1933 .

5 Cette famille de fossores figure sur l'inscription du cimetière indiquée par $O$. Marucchi, loc. cit., et sur une autre connue par une copie d'A. Bosio, Roma Sotterranea, ed. posthume, Rome, 1632, chap. 50. 
78.

Le même (?) PROCLLS figure sur une inscription du même cimetière en compagnie de IOANNis et ILCILIJANIS, fossores enx aussi (?)

VIA SAIARIA

Giordani

79. avant 337?: AEI. ALXANON, fosor, construit une tombe pour lui et sa femme

80. date: ?: T[R]OFIML's (peinture)

\section{Priscille}

81. avant 337: VIBICS, fossor, épitaphe

Avant le milieu du IVe siècle? 2:

82.

82 bis

82 ter

83.

84.

85.

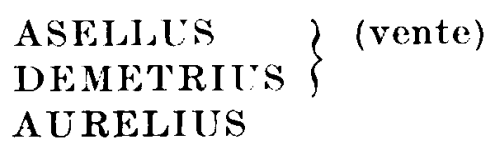

CANDIDUS

HERTILELS FRIGIANUS RIFLS ICCILIUS, fossor, épitaphe PATERNUS, fossor, épitaphe
Wilpert ${ }^{1}$, pl. IV, 2.

Boldetti, p. 65.

Marucchi, p. 407.

RAO, 1931, p. 230.

Boldetti, p. 53.

Marini, Vatican latin, 9072 , fo 1056.

ibid., fo 2754.

Muratori, 1969, 8.

Muratori, $1969,6$. Muratori, 1969, 7 .

Via Fla MINIA

Saint-Valentin

Après 337:

86.

87.

88.
ACILLINLS, fossor (vente)

LIBERLS, fossor (vente)

Le même (?) LIBERUS à propos d'une vente de tombe
Marucchi, p. 607. Diehl, $3757 \mathrm{~B}$.

Marucchi, p. 607.

1 J. Wilpert, Die Katakombengemalde und ihre alten Kopien, Fribourg, 1891.

2 I a datation des inscriptions de fossores trouvées à Priscille, toutes connues par des copies anciennes, est difficile. Le formulaire invite à les placer à une date assez haute, probablement antérieure au milieu du IVe siècle. Certaines peuvent cependant avoir été rédigées entre la mort de Constantin et l'avènement du Pape Damase.

C"est pourquoi je renonce ici à appliquer strictement mon système de classement.

3 Herculeus et Frigianus, fossores dédient l'épitaphe à leur frère Rufus, mort à 31 ans, et fossor lui aussi. 
ISCRIPTIONS D'ORIGIXE INCONNUE OU INCERTAINE

Avant $337:$

89.

90.

91.

92. date:?

Après 337:

93. $a^{\circ} 338^{1}$ :

94. a 404 :

95. $a^{0} 395$ ? $465 ?^{3}$ :

96.

97.

98.

99.

100.

101.

102.

103.

104.

105.

106.

107.

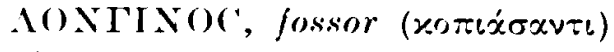
(épitaphe)

TERENTI's- fossor, epitaphe

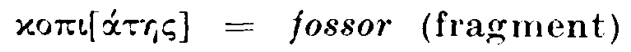

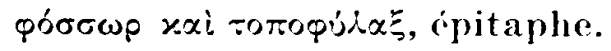

IANIARILS, fossor (vente)

$V[\ldots . .$.$] , fossor (vente) { }^{2}$

[...]CS, fossur (vente pour 3 sous) AQIIIINCS, fossor (vente)

AlRENTINIS, fossor (vente)

('[...] (vente) ${ }^{4}$

CEMENT[ARILS? (vente) ${ }^{5}$

FEIIX, fossor, épitaphe.

FELIX, fossor, épitaphe.

GALDENTILS, fossor (vente)

HILARIS
LALRENTIIS $\left\{\begin{array}{l}\text { fossores, vente } \\ \text { pour 500 folles }\end{array}\right.$

SEVERIS

Le même (?) SEVERL's, nommé sur l'épitaphe de sa femme?.

IIIAA R'S, fossor (vente)

LEONTILS, fossor (vente)

[MA]XIMIN[CS] (vente?) ${ }^{8}$. $\begin{array}{ll}\text { ICUR, NS, } & \mathrm{I}, 4021 . \\ \text { ICUR, NS, } & \mathrm{I}, 1727 . \\ \text { ICUR, NS, } & \mathrm{I}, 1395 . \\ \text { ICER, NS, } & \mathrm{I}, 4072 .\end{array}$

IOUR, NS, I, 1647.

IVUR, NS, I, 1402.

ICUR, NS, I, 3240.

ICUR, NS, I, 1190.

ICUR, NS, I, 1642.

ICUR, NS, V, 15365.

$I C U R, N S, \quad \mathrm{I}, 1288$.

ICUR, NS, I, 1606.

$I C U R, N S, \quad \mathrm{I}, 377$.

ICUR, NS, I, 2607.

ICLR, NS, I, 1282.

1 I a date consulaire est très mutilée. Ie premier éditeur, A. Silvagni, proposait de comprendre 488; en donnant une meilleure interprétation des lettres conservées, A. Ferrua, $P A R A(R), 1963-1964$, p. 115 , propose 338.

2 O. Marrucchi, $N B A C, 1900$, p. 137 rapproche cette inscription où le nom du fossor a été rasuré de celle de Cyriaque, no 65 de mon catalogue, où le nom du fossor est mutilé, et l'attribue donc à Saint-I aurent. Le rapprochement ne me paraît pas entièrement probant, et, dans tous les cas, il est pour le moins douteux que le nom du fossor ait été le même sur les deux inscriptions.

3 L'inscription fut trouvée sur la via Tiburtina. Avec l'éditeur, il faut penser que la date la plus tardive est la plus probable.

${ }^{4}$ L'inscription provient de la zone de la via Appia.

5 Ce nom peut être aussi celui du métier du défunt.

- Hilarus, fossor vend la tombe en présence de fossor Severus et de Laurentius, qui est sans doute fossor lui aussi.

? I'inscription est très mutilée. Il semble pourtant que la femme du fossor Severus est inhumée en même temps que la femme d'un certain Hilarus. S'agitil des mêmes personnages que ceux qui sont mentionnés sur l'inscription précédente?

\& Le fragment est très mutilé, et toute restitution est aventureuse: il n'est pas sûr que le nom restitué, Maximinus, soit celui du vendeur. 
108.

SLSTCS, fossor (vente pour 3 sous un tiers) ${ }^{1}$

109. BAL[ENTINLS] (vente) ${ }^{2}$

ICUR, NS, I, 2223. ICUR, NS, I, 117. ... NLS, fossor, témoin à une vente? fossor, sur une inscription de vente fragmentaire. ICUR, NS, I, 1334.

111.

112. fossor auquel s'adresse une épitaphe: fossor, vide ne fodias... ${ }^{3}$ Diehl,

La société des fossores. Etude onomastique ${ }^{4}$.

Avant comme après 337, les fossores suivent l'évolution de l'onomastique de leur temps et portent des noms uniques. On notera seulement quatre exceptions: avant 337, C. Picentius Formonsus, 37 , porte les tria nomina, ce qui suffit à attester l'ancienneté de l'inscription, car on ne trouve que rarement dans l'épigraphie chrétienne de Rome des inscriptions portant les tria nomina après la paix de l'Eglise (I. Kaianto, dans Sylloge, p. 43); avant 337 aussi, Ael. Auxanon 79, et après 337 , si l'on admet l'exactitude de nom classement sur ce point, Aur. Constantinus et Aur. Biaturinus, 21, portent les duo nomina: dans ces trois derniers cas, le gentilicium est d'une si grande banalité qu'il a été écrit en abrégé, selon une habitude courante dès la fin de l'époque impériale (I. Kaianto, ibid.).

Mais, pour les reste, les noms des fossores attestés avant et après 337 présentent entre eux des différences qui sont plus que des nuances: aussi

1 On pourrait songer à une localisation sur la via 'Tiburtina, si l'on rapproche ce nom du no $75^{\text {bis. }}$.

2 L'éditeur restitue praepositus sur cette inscription lacunaire. Le formulaire the fait préférer la restitution fossor, car l’inscription est sans doute des IVe-Ve siècles.

3 L'attribution est douteuse, cette formule de précaution nell'impliquant pas nécessairement l'intervention effective d'un fossor.

4 comme toute étude onomastique, celle-ci doit beaucoup à l'ensemble des travaux d'I. Kaianto, dont j'indique ici les références et, entre parenthèses, les abréviations que j'utiliserai : commentaire onomastique, dans Sylloge Inscriptionum Christianarum Musei Vaticani, de H. Zilliacus (= sylloge); Onomastic Studies in the early christian inscriptions of Rome and Carthage (=On. Studies), vol. I, 2 et II des Acta Instituti Romani F'inlandiae, Helsinki, 1963, et The Latin Cognomina ( $=$ Cognomina), vol. XXXVII, 2 des Commentationes IIumanarum Litterarum de la Societas Scientiarum Fennica, Helsinki, 1965.

5 Je donnerai désormais pour les fossores la forme du nom telle qu'elle a été restituée par l'éditeur, quand il y a eu restitution. On se reportera à mon catalogue pour juger de limportance de ces restitutions. 
doit-on les étudier séparément, en veillant toutefois à ne pas trop accuser les oppositions, car tout classement chronologique comporte ses incertitudes.

Avant 337, un nom sur cinq environ est grec, ou d'origine grecque: Alexander, 35, 36, Auxanon, 79, Calligonus, 77, Trofimus, 80. La proportion est moindre que celle relevée dans les inscriptions chrétiennes conservées au Musée Vatican, (27,5\%: I. Kaianto, dans Sylloge, p. 49), mais reste comparable, et la diffrence peut s'expliquer par un accident statistique dû au matériel réduit de mon enquête.

A l'exception de Debestus, 76, qui est un hapax (Thesaurus L. L., Onomasticon, s.v., col. 66), la plupart des noms appartiennent au répertoire courant des noms latins, Felix, 1, Festus, 60, Erculius, 61, Iunius, 20, 27, Leo, 50, Mov'ívos, 89, Lucillianus, 78, Maius, 77, Proclus, 77, 78, Sergius, 20, Terentius, 90, Tigrinianus, 61. Si certains, Felix, Iunius, Maius, Proclus, sont des noms extrêmement répandus, et connus depuis longtemps, d'autres traduisent l'engouement de la basse antiquité pour des cognomina dérivés d'autres cognomina par suffixation: ainsi, selon I. Kaianto, Cognomina, p. 329, Tigrinianus est un hapax, sans signification chrétienne pourtant.

Le fait marquant est précisément la rareté des noms chrétiens, qui ne sont représentés que par deux noms tirés de la Bible (soit moins de $10 \%$ ), Ioannes, 78, et Petrus, 62 alors que l'on aura noté dans la rubrique des cognomina latins la présence d'un nom mythologique, Erculius, 61.

Au total, le répertoire des noms dans la première période est donc d'une grande banalité: les fossores semblent recrutés sur place, ou provenir d'une immigration proche, et porter des noms peu marqués par la religion à laquelle ils appartiennent.

Après 337 se dessinent les traces d'une évolution. Ainsi, la proportion des noms grees est en sensible diminution: moins d'un nom sur dix seulement, Anthiochus, ou Athanasius, 66, Demetrius, 82, Diogenes, 22, Euse [...], 51, Iconius, 31, du nom de la ville de Cappadoce? (I. Kaianto, On. Studies, p. 85), Leontius, 106 (j'ai suivi I. Kaianto, On. Studies, p. 55 pour le classement de ce nom), Musicus, 51. Avec I. Kaianto, dans Sylloge, p. 49 , on peut tenter d'expliquer cette diminution par la vogue de romanisation des cognomina qui touche souvent les familles immigrées dès la seconde génération, au moment où la diminution de l'esclavage venu d'Orient entraîne un affaiblissement de l'onomastique d'origine grecque.

Inversement, un certain nombre de noms indiquent plus ou moins clairement une immigration récente: Apro, 67, quoique connu à Rome (I. Kaianto, Cognomina, p. 325) pourrait être originaire de Norique (cf. CIL, III, 5285); Burdo, 9, d'Afrique (Cognomina, p. 326); Calevius, 55 
de Byzacène (Thesaurus L.L., Onomasticon, s.v., col. 80); Fortunio, 51, quoiqu'attesté à Rome, vient peut-être de Bétique ou de Dalmatie (Cognomina, p. 273); Mulia, 14, de Gaule (Ibid., p. 323); Paternus, 85, a près de deux chances sur trois d'être gaulois ou espagnol (Ibid., p. 85); Safargius, 73, peut être africain selon Diehl, Index, I, s.v. et Sicu, 56, africain lui aussi (Cognomina, p. 264).

A l'exception d'Arintatme, 59, visiblement corrompu, de Muscorutio, 9, 10, que je renonce à interpréter et de Micinus, 9, attesté à Rome, mais peut-être étranger (bien que I. Kaianto, Cognomina, p. 348, n'en donne pas l'origine), les autres noms sont des plus communs, et sacrifient souvent à la mode de la dérivation par suffixation, ainsi Astaerius, 29, Celerinus, 63, Constantinus, 21, Exuperantius, 13, Florentius, 48, Gaudentius, 102, etc.

A côté de ce répertoire banal, les noms chrétiens sont en nette augmentation. Avec I. Kaianto, il faut renoncer à compter parmi eux les prétendus "noms d'humilité ", comme Proiectus(?), 15, ou Proiecticius 7, 8, qui, bien que fortement représentés chez les chrétiens, sacrifient à une mode générale de l'antiquité tardive ${ }^{1}$; de même, Gaudentius, 102, ne traduit pas une joie seulement religieuse (I. Kaianto, On. Studies, p. 67), mais on peut en revanche compter dans le matériel près de $12 \%$ de noms chrétiens qui peuvent se répartir ainsi:

- noms incluant le nom de Dieu: Adeodatus, 5, 11, et Quodvuldeus, 47, "noms-phrases " étrangers à l'onomastique gréco-latine traditionnelle, et d'origine africaine (I. Kaianto, dans Sylloge, p. 69): faut-il voir ici l'indice d'une immigration? ou plus probablement le succès d'une mode?

- noms exprimant des idées chrétiennes: (ici, résurrection et rédemption) Anastasius, 66, et Redemptus, 4 (cf. On. Studies, p. 110-111);

- noms tirés de la Bible: Andreas, 12 (mais la restitution est hypothétique), Petrus 46;

- noms de martyrs: Laurentius, 40. Le nom porte une suffixation à la mode, mais son succès chez les chrétiens est certainement dû au martyr (On. Studies, p. 99); en revanche, il peut être douteux que Epolytus, 3 (cf. On. Studies, p. 99) et surtout Sustus, $75^{\text {b1s }}$ et 108, aient été choisis en référence aux célèbres martyrs.

Malgré tout, la recension des noms de cette seconde période montre que, si l'origine géographique des fossores s'est diversifiée, la christianisa-

1 A ce sujet, voir I. Kaianto, On the problem of names of humilty in early Christian epigraphy, dans Arctos, NS, III, 1962, p. 45-53. 
tion de l'onomastique demeure timide: bien que leurs fonctions les mettent en contact plus étroit avec les cleres que les simples fidèles, les fossores ne semblent avoir subi en ce domaine aucune influence déterminante, et leurs noms semblent plus près de ceux du gros de la communauté des fidèles que de ceux du clergé ${ }^{1}$.

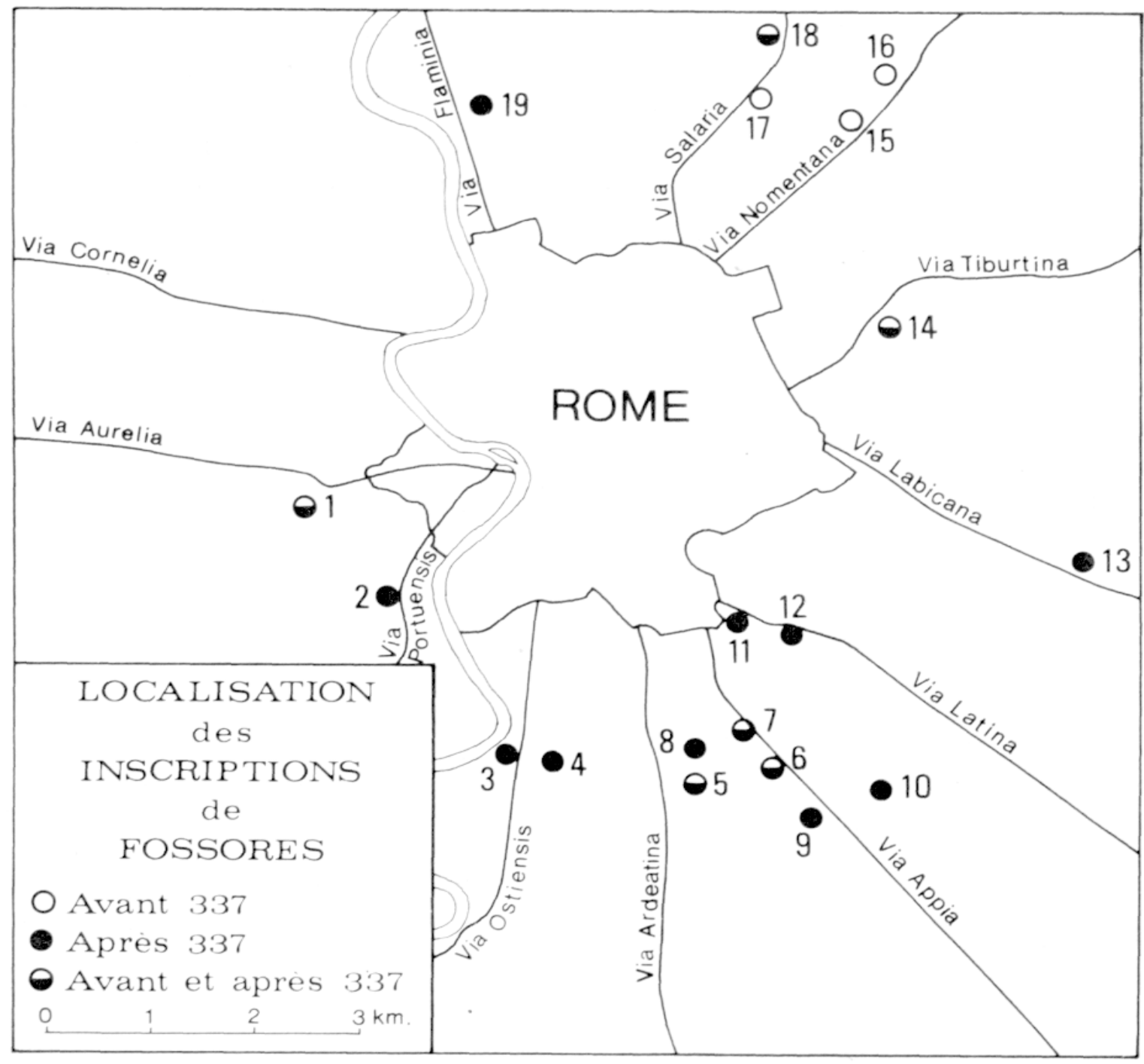

Fig. 2 - CARTE DE localisation DES INSCRIPTIONS DE "Fossores". 1. Saint-Pancrace. 2. Pontien. 3. Area Lucinae. 4. Commodille. 5. Domitille. 6. Callixte. 7. Marc et Marcellin. 8. Cimetière près de l'Ardéatine. 9. SaintSébastien. 10. Prétextat. 11. Gordien. 12. Apronianus. 13. Pierre et Marcellin. 14. Saint-Laurent. 15. Sainte-Agnès. 16. Cimetière Maius. 17. Cimetière des Giordani. 18. Priscille. 19. Saint-Valentin.

1 C'est l'impression que donne un examen rapide des noms des clercs romains dans la liste incomplète que donnent les I.L.C.V. de F. Diehl. Le ca- 
Le domaine d'activité des fossores.

On peut dresser une carte des inhumations ou des interventions des fossores (Fig. 2), et on voit au premier regard que tous les cimetières importants y figurent, à deux exceptions près cependant: les grandes memoriae apostoliques de Saint-Pierre et de Saint-Paul qui, sur ce point aussi, semblent avoir bénćficié d'un traitement particulier.

Sans doute pourrait-on tenter une description plus précise, en se fondant sur le découpage chronologique. L'entreprise pourtant est difficile, car la documentation reste lacunaire (les inscriptions étant connues au hasard des trouvailles et de l'avancement de leur publication), et l'épigraphie ne peut pas toujours à elle seule donner de la réalité historique une image suffisamment ficlèle.

Un exemple le montrera clairement: on remarquera qu'avant la mort de Constantin, ce sont essentiellement les cimetières de la région nord, Cyriaque, Sainte-Agnès, le cimetière Maius et celui des Giordani qui fournissent des documents, auxquels doivent s'ajouter ceux fournis par la catacombe de Priscille qui, tous, paraissent antérieurs à la moitié du IVe siècle, tandis que le cimetière de Callixte, par exemple, n'est représenté que par une inscription, 27, et que celui de Pierre et Marcellin sur la via Labicana semble totalement absent (sauf peut-être si le no 58 appartient à cette période). Or, Callixte, je l'ai dit, fournit des représentations peintes de fossores fort anciennes, et Pierre et Marcellin est la catacombe la plus riche en re domaine; or, si le problème de la datation des peintures de ce cimetière est loin d'être résolu, un accord général semble les placer pour l'essentiel à l'époque constantinienne ', ou même avant. Ici donc, le témoignage iconographique doit corriger celui de l'épigraphie.

Quand les documents sont plus nombreux, à partir du milieu du IVe siècle, on voit apparaître en pleine lumière, comme on pouvait s'y attendre, la plupart des cimetières où la présence de tombes de martyrs

talogue prosopographique du clergé romain que publiera ('h. Piétri dans sa thèse Roma christiana permettra d'intéressants rapprochements et une comparaison chiffrée.

1 On se reportera, pour la reproduction des peintures de fossores de la catacombe de Pierre et Marcellin à J. Wilpert, Le pitture delle catacombe romane, Rome, 1903, pl. 48, 59, 65,3;107,2;112,5; $113 ; R^{\prime} A C, 1932$, tables 2 et 12 ; P. Testini, Archeologia cristiana, Rome, 1958, pl. I; pour la datation des peintures du cimetière aux relations de J. Kollwitz et $\mathrm{L}$. de Bruyne sur la peinture constantinienne dans Akten des VII Internationalen Kongresses für christliche Archaölogie, Trêves, 196.), Rome-Berlin, 1969, p. 44 sq. 
entraîne d'importants travaux d'embellissement et de restauration, l'édifieation de retrosanctos et la multiplication des contrats de vente: les cimetières de Domitille, Commodille, Callixte, Cyriaque, et même la petite nécropole de Saint-Valentin sont en pleine activité, et l'épigraphie nous montre des équipes entières de fossores au travail pour répondre à la demande des fidèles.

Pourtant, des anomalies demeurent: comment expliquer, par exemple, l'importance inégale de la documentation pour la série des cimetières où Constantin avait fait édifier des basilicae 1 ? Tandis que le cimetière de Cyriaque, près de Saint-Laurent fournit treize inscriptions, nos 63 à 75, Saint-Sébastien n'en a que quatre, nos 47-49 et 51, Pierre et Marcellin, une ou deux, nos 58-59, et Sainte-Agnès, aucune pour cette période. Dans le cas de Pierre et Marcellin, comme dans celui de Saint-Sébastien, une suggestion du Père $\Lambda$. Ferrua pourrait fournir un élément de réponse: pour lui, le succès de la basilica constinienne, destinée elle aussi à l'inhumation, aurait éclipsé celui de la catacombe ${ }^{2}$; à Pierre et Marcellin la perte du monument constantinien dont les fouilles n'ont révélé que de pauvres vestiges ${ }^{3}$, comme à Saint-Sébastien de profondes transformations pourraient expliquer le silence, ou du moins la pauvreté de l'épigraphie. Peutêtre pourrait-on avancer une explication similaire pour le cas moins net de Sainte- $\Lambda$ gnès? Le doute reste permis, et ce simple exemple sert surtout à montrer qu'ici comme ailleurs la maniement de l'argument a silentio pourrait conduire à de graves erreurs. Aussi est-il plus prudent, dans l'état actuel de la recherche, de laisser la question ouverte.

\section{L'organisation du travail.}

Ici, l'interprétation des documents est beaucoup moins problématique, et les renseignements qu'ils fournissent permettent assez bien de décrire les conditions d'exploitation des cimetières ${ }^{4}$.

1 Sur ces édifices, voir notamment $R$. Krautheimer, Mensa-coemeteriummartyrium, dans Cahiers Archéologiques, XI, 1961, p. 15 sq.

2 A. Ferrua, Una nuova regione della catacomba dei SS. Harcellino e Pietro (continuazione), dans $R A C, 46,1970$, p. 83.

3 sur ces fouilles, voir F.-W. Idehmann et A. Tschira, Mas Mausoleum der liaiserin Helena und die Basilika der Heiligen Marcellinus und Petrus an der Via Labicana vor Rom, dans $J D A I, 72,1957$, p. $44 \mathrm{sq}$.

- Dans une étude essentiellement fondée sur l'utilisation de documents épigraphiques, je laisse de côté les éléments proprement techniques du travail des catacombes pour me limiter à la seule organisation d'ensemble de ce travail. 
L'impression qui s'en dégage est celle d'une grande diversité, ou mieux d'une grande souplesse d'organisation. A examiner les documents épigraphiques en effet, il ne semble pas qu'il y ait eu de règle générale, mais plutôt des solutions très variées, adaptées à la nature des cimetières et aux problèmes spécifiques qu'ils pouvaient poser.

Ainsi, on trouve côte à côte dans le même cimetière des inscriptions où un seul fossor vend la tombe qu'il a aménagée, et, dans une minorité de cas, des textes où des équipes de fossores vendent collectivement le produit de leur travail. Pourtant, le jeu des associations demeure très libre: à Commodille, en 426, le fossor Muscorutio s'est associé à ses collègues Burdo et Micinus pour la vente d'une tombe, 9, mais vend seul dans le même cimetière un bisomus, 10. Peut-être l'association ne durait-elle dans certains cas que le temps d'une tâche précise? peut-être aussi la mort de certains participants l'avait-elle détruite?

Il est clair en tout cas que la présence d'équipes de fossores est sûre dans au moins un cimetière sur trois, et il ne me paraît guère douteux qu'elles étaient plus nombreuses que ne le laisse voir la documentation. Ainsi, on voit des équipes à l'œuvre à Saint-Pancrace, 2, dans le cas de Bictorinus et Exuperus, collega ipsi; à Commodille, dans l'exemple déjà cité, 9; à Domitille, 21, où Aur. Constantinus seconde son collègue Aur. Biatorinus, sans doute illettré, dans la rédaction d'un contrat de vente; à Saint-Sébastien, 51, où Musicus a signé son ouvre et celle de ses compagnons de travail; à Prétextat, 54, où quatre fossores seraient associés dans une vente si l'on accepte l'interprétation que je donne de cette inscription; à Saint-Laurent, 66, où Anastasius (ou Athanasius) et Anthiochus, mais aussi Apro et Biator, 67, s'associent pour la vente de tombes; à Priscille enfin, où l'on peut citer l'association d'Asellus et Demetrius, 82.

Sans qu'on en ait de témoignage parfaitement clair, les liens de famille pouvaient peut-être à l'occasion servir d'ossature à de telles équipes: Sergius et Iunius, réunis dans la tombe à Domitille, 20, étaientils parents ou compagnons de travail? Il existe en tout cas au moins deux familles de fossores dans la documentation: Herculeus et Frigianus à Priscille, 83, sont tous deux fossores, et dédient une tombe à leur frère Rufus, fossor lui aussi, et les deux inscriptions très mutilées du cimetière Maius, 77 et 78, gardent la trace d'une véritable "dynastie " de fossores de ce cimetière, mais leurs textes sont si lacunaires ou si corrompus que je renonce à tirer au clair leur arbre généalogique.

Des équipes de fossores, qui pouvaient se former ou se défaire selon le travail demandé, étaient donc à l'œuvre dans les cimetières, et trois indices au moins peuvent faire penser qu'elles se chargeaient à l'occasion plus particulièrement de telle ou telle partie de la nécropole: ainsi, autour des années 400 , on voit le même Proiecticius vendre deux tombes très 
voisines à Domitille, 7 et 8; en 405, à Saint-Laurent, 65, pour la vente d'une tombe dans la partie droite de l'escalier descendant à la crypte historique, le fossor, dont le nom a été rasuré, est désigné par les termes fossor... l]oci ipsius; quelques années plus tard ' l'acheteur d'une tombe de l'escalier (discindentib[... dit l'inscription mutilée, 75) ne mentionne pas le nom du fossor qui la lui vend, sans doute parce qu'il était encore le fossor loci, chargé de la crypte et de ses abords.

Malgré la netteté de ces exemples, il serait faux d'imaginer une séparation tranchée entre régions d'une catacombe, ou même entre cimetière souterrain et nécropole sub divo. Sans doute, seuls de tous leurs collègues, un fossor de Callixte ${ }^{2}$, et Trofimus, 80 (Fig. 3) sont-ils représen-

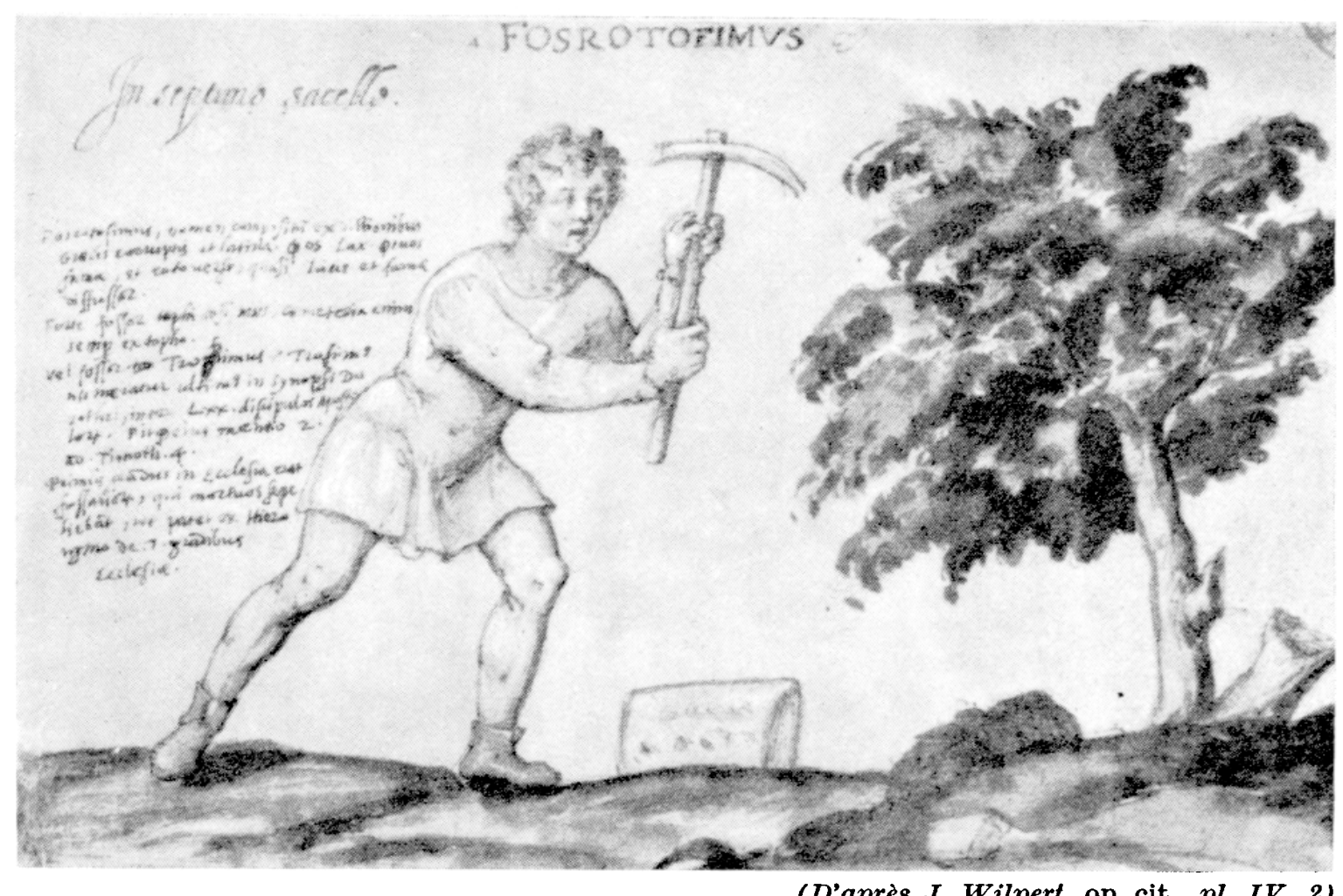

Fig. 3 - Cimetière des Giordani. Le "Fossor" T[R]ofimus travaillant à la surface. Copie ancienne de Ciacconio, Vat. IaAt., 5409, fo 13: L'ORIGINAL EST PERDU.

1 Peut-être vers 410-430, à en juger à la fois par le formulaire, costat me emisse..., qui est attesté pour la première fois, 9 , en 426 , et par le nom de l'acheteur, qui porte les duo nomina, qui deviennent fort rares après 410 (I. Kaianto, On. Studies, p. 43).

2 G.-B. de Rossi, R.S., tavole, II, pl. XVIII. 
tés sur des peintures travaillant à la surface; sans doute aussi Felix, 44, indique-t-il dans un contrat qu'il a édifié une sépulture hypéthère, locus sub teglata, près de la basilique du Pape Marc, mais rien n'indique que le travail de ces ouvriers ait été limité à ces domaines particuliers et, inversement, à Sainte-Agnès, Debestus, 76, précise qu'il a travaillé dans

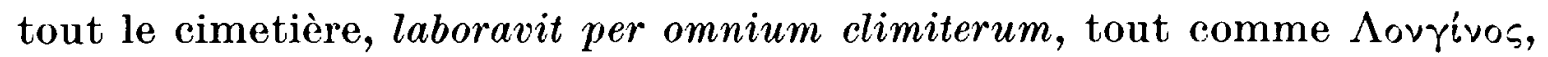

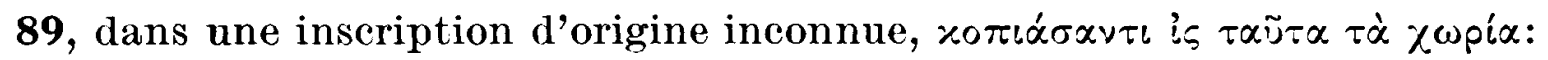
aussi est-il certain qu'ici encore la plus grande souplesse était de règle, et que les mêmes hommes, ou les mêmes équipes, étaient employés, en fonction des besoins, dans des endroits différents.

Rien n'indique très précisément en revanche comment était organisé le métier et s'il existait une hiérarchie des fossores. On a quelquefois essayé de le suggérer à partir de la peinture de IDiogenes, $22{ }^{1}$ : sans doute cette peinture, malheureusement perdue aujourd'hui, mais connue par une copie ancienne ${ }^{2}$ (Fig. 4) tranche-t-elle nettement par l'abondance et la netteté des outils représentés comme par son sujet (c'est le portrait d'un fossor et non une représentation de fossor) sur les autres peintures de fossores, figurés isolément ou par deux, vêtus de la tunica discinta, munis simplement du piccone et souvent d'une lampe. Mais, au lieu de voir dans la peinture de Diogenes celle d'un chef fossor, on peut aussi y trouver sans doute un témoignage un peu naïf d'autosatisfaction de la part d'un fossor arrivé à une certaine réussite par la pratique de son métier.

D'ailleurs, les témoignages épigraphiques ne plaident guère en faveur de l'existence d'une hiérarchie: si à Saint-Sébastien, 51, Musicus signe avec ses ouvriers, mais avant eux, cum suis laborantibus, on notera que la vocabulaire qu'il emploie fait plus penser à des manouvres ou des aides qu'à de véritables fossores, et, dans les autres cas, c'est incontestablement sur la base d'une stricte égalité que se placent les rapports entre fossores. La vente de l'inscription no 2 est en effet effectuée par deux "collègues ", et lorsqu'on voit à Commodille, 19, tous les fossores figurer à une vente comme témoins, rien n'indique entre eux la moindre préséance.

Ce trait concorde parfaitement avec l'organisation de leur travail, qui reste le plus souvent individuel. Du reste, la satisfaction d'Iconius, 31, heureux de noter par un graffito qu'il a achevé en dix jour's un cubiculum, comme celle d'Ael. Auxanon, 79, qui précise avoir creusé suis manibus sa tombe et celle de sa femme ne sont-elles pas d'excellents

1 G.-13. de Rossi, R.S., III, p. 539.

2 A. Boldetti, op. cit., p. 60. 


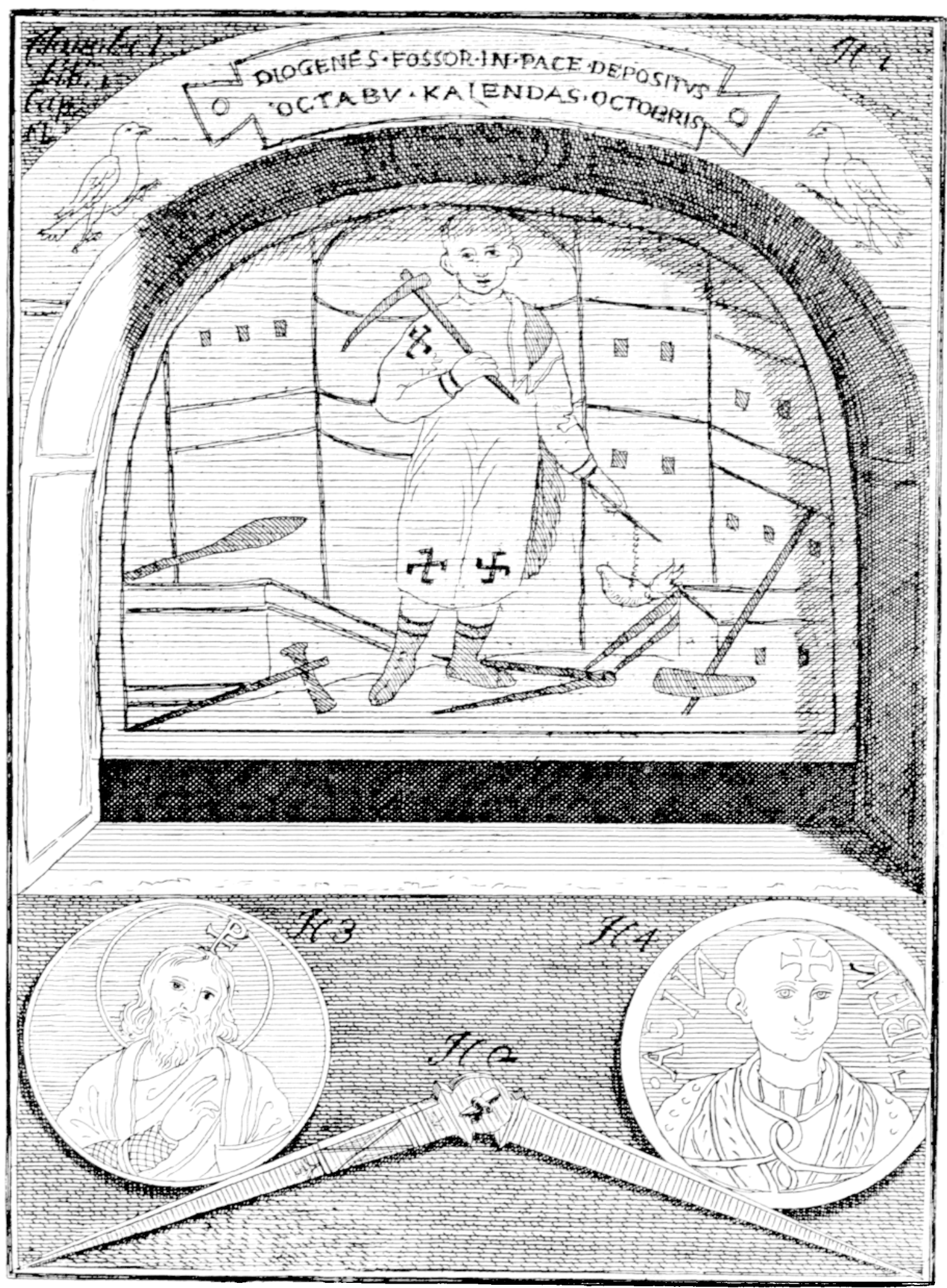

(D'après Boldetti, op. cit., p. 60)

Fig. 4 - Cimetière de Domithl.e. Peinture, aujourdinu perdee, REPRESENTANT LE "FOSSOR" DIOGENES ET SES OUTILS DE TRAVAIL. 
témoignages de l'indépendance, voire de l'individualisme, de cette petite société d'artisans besogneux que l'on aperçoit en plaine lumière au moment de l'établissement des contrats de vente?

Les contrats de vente passés par les fossores.

Souplesse et diversité dominent ici encore, car des rédactions différentes coexistent dans un même cimetière, et certaines formules continuent leur carrière même quand d'autres, plus élaborées, apparaissent. Un trait domine pourtant: la précision se fait plus grande avec le temps, et la forme devient plus nettement juridique.

Emere et comparare sont les deux verbes les plus utilisés dans le formulaire, mais avant de disparaître, le vieux verbe de dédicace facere s'associe à trois reprises à emere, 25, 43, 59.

Dans la plus ancienne inscription datée sûrement, 93 ( $a^{\circ} 338$ ), emere est employé à la première personne du singulier, ego himi locum a fossore, et l'on trouve d'autres exemples de cet emploi, avec emere, 42 et comparare, 5, 13. Mais dans le grande majorité des cas l'entraînement du formulaire des autres inseriptions funéraires conduit à utiliser la troisième personne du singulier ou du pluriel: comparavit, 41, 110; emit, 14, 17, 32, 71, 106, avec des variantes, soit vivus/i ou se vivus/i comparavit/erunt, 64 (a 403 ), 95 (a 465 plutôt que 395), 18, 111, se vivi emerunt, 63 (a 400), 11, 15, 26, 57, 97, soit sibi comparavit, 2, soit la combinaison de ces deux formules, se vivi locum sibi emerunt, 19, 67, 102, ou se vivus sibi locum comparavit, 65 (a 405 ), 10 (vers 426), 70, 72.

L'apparente banalité de ce premier groupe de formules qui sont utilisées tout au long de la période où les contrats sont connus ne doit pas masquer leur profonde originalité par rapport au gros des épitaphes qui leur ressemblent tant: avec elles, sur la tombe, ce n'est plus un hommage au mort qui figure, mais un véritable titre cle propriété.

On peut même se demander si les exemplaires datés indiquent le jour du décès ou celui du contrat et, à la vérité, deux inscriptions seulement semblent indiquer presque à coup sûr le jour de la sépulture: le cas est certain pour le no 95 qui porte après la mention de l'achat la formule de]posita...; pour le no 63, emit Soteres se viva et marito suo Vernaculo compari suo emit a Celerino fos, on doit comprendre, semble-t-il, que Soteres a acquis au jour du décès de son mari un bisomus qui lui servirait également à sa mort.

Un second type de formules est plus directement marqué encore par le style des contrats. Déjà l'inscription no 49 emprunte au langage juridique: ego comparavi... ego s(upra) s(criptus) vivus; plus net encore 
est l'exemple du no 6 (a 380 ), me vibo s(cripsi) comparasse locum; directement tirées du langage des affaires enfin sont les formules costat me (ou se ou nos) emisse locum, 9 (a 426), 75, 108.

Un troisième groupe apparaît concurremment qui, au lieu de résumer les contrats de vente, en indique les effets: il s'agit des inscriptions portant en tête le terme locus, suivi du nom du propriétaire au génitif, et mentionnant ensuite l'achat et le nom du vendeur par des formules du type: locus $N$. comparatus . ., 7 (a 398), locus $N$. emptus . ., 45 (a 403), $3\left(a^{\circ} 430\right), 23,43,56,103$; ou, avec un verbe à la forme personnelle, locus $N$. comparavit/erunt, 8 (vers 398), 66, 99, 109, ou une proposition relative, locus $N$. quem emit, 28 (a 378 ). On notera dans ce groupe une formule rare, 98, locus Bonifat[...] est adquisit a $C[\ldots]$.

Dans tous ces cas, c'est l'acheteur qui est l'agent, et l'on ne trouve que deux exemples où c'est le vendeur qui s'exprime: vendidit trisomum, 55 ( a $^{\circ} 400$ ), et scripsi pro (...) eum vendidisse, 21. Il n'y a pas lieu de s'en étonner: de telles formules sont une garantie pour le client, et c'est certainement à sa demande qu'elles sont rédigées; aussi est-il normal qu'il y tienne la place essentielle.

La réalité matérielle des contrats de vente n'est guère niable, car le rapprochement que de Rossi a tenté entre ce dernier type de formules et de véritables contrats retrouvés à Pompei est parfaitement convaincant ${ }^{1}$. Peut-être même conservait-on une copie de l'acte, ou son original, dans les archives de la catacombe, si l'on en croit une inscription d'origine inconnue qui appelle à se rendre au cimetière en cas de contestation (le simple résumé donné sur la tombe ne suffisait done pas toujours!): locum Vincenti quem comparavit cum suis. Si qui voluerit requirere veniat in cle[meterium (ICUR, NS, I, 3868).

Encore fallait-il que tous les termes du contrat fussent scrupuleusement respectés: aussi voit-on apparaître par précaution des clauses plus précises. Ainsi, certaines inscriptions indiquent clairement la capacité de la tombe, ou le nom de ceux qui sont destinés à y être enterrés. On ne manquera pas d'être frappé par la forte proportion, près de $40 \%$, des inscriptions du matériel retenu pour cette étude indiquant expréssement des achats de bisomus, trisomus, etc., tandis qu'un tiers seulement des contrats sont le fait de personnes seules, les autres $27 \%$ étant des actes passés par deux personnes et correspondant sans doute à des tombes à deux places: une telle proportion ne peut s'expliquer que par le souci de garantir une sépulture assurée au dernier survivant.

1 G.-B. de Rossi, R.S., III, p. 546. 
Par précaution aussi, certains contrats sont passés en présence de témoins: à Commodille, 11, la vente est effectuée sub praesentia sancti Maximi presbyteri, mais, ici encore, il n'y a pas de règle fixe, puisque dans le même cimetière ce sont tous les fossores qui sont témoins d'une autre vente, 19, presentis omnis fossores; une clause identique figure sur une inscription d'origine inconnue, 103, où Severus et Laurentius sont témoins d'une vente faite par leur collègue, le fossor Hilarus; enfin, l'inscription 110, très mutilée, porte le mot presentem, sans qu'on puisse connaître ni le nom, ni la qualité du témoin, car le fossore de la ligne suivante a toute chance, par sa forme grammaticale, de se rapporter au vendeur plutôt qu'au témoin.

Une autre précision figure à l'occasion sur les inscriptions, celle de la localisation de la tombe: à Commodille en 426, l'emplacement est situé ante domna Emerita, 9; à Domitille, 25, au cymtera Vapiatina; à Callixte, 32, ad sanctum Cornelium; dans le cimetière près de la voie Ardéatine, in crupta, 42, et dans l'escalier d'accès, gradu quintu discindentibus, 43; à Balbine, 44, in Balbinis locum sub teglata; à Saint-Sébastien, 49, ad lumenarem; à Prétextat, 54, in cimi]tero Pretextat $[$ ad sanctum Quiri]nu martore; à Saint-Laurent, en 405, no 65, ad mesa beati martiris Laurenti descindentib in cripta parte dextra, et, 75, sans doute dans le même escalier, discindentib $[. .$. , et, 67 , in crupta noba retro sanctos.

I)ans dix cas sur onze, la précision topographique est grande, et, dans la quasi-totalité des cas, la tombe est localisée à proximité immédiate d'un lieu vénéré de la catacombe (crypte, retro-sanctos, et leurs escaliers d'accès) ou d'une basilique du cimetière sub divo.

Un sondage rapide effectué sur les inscriptions indiquant une vente, sans préciser le nom du vendeur, conduit au même résultat ${ }^{2}$. C'est l'affluence autour des tombes des martyrs, et la concurrence qu'elle crée, qui poussent sans aucun doute l'acheteur à préciser ainsi ses titres de propriété, dans la crainte de les voir bafoués, et la présence dans le matériel conservé de nombreux remplois ${ }^{2}$ montre à quel point ces inquićtudes etaient foncées.

1 A commodille, par exemple, cest près de la crypte historique que se trouve le plus grand nombre dinscriptions de ce type qui soient exactement localisées, et le phénomène est identique à Jomitille.

I) autre part, on trouvera dautres inseriptions mentionnant precisément le lieu de sépulture, et qui n'entraient pas dans les limites de mon enquête, dans P. 'Testini, Le catacombe e gli antichi cimiteri cristiani in lioma, Rome, 1966, p. 204-206.

2 Pour se limiter à des exemples pris dans le matériel utilisé pour cette etude, on peut citer les inscriptions remployées ICUR, NS, I, 2664, II, 5168, 
Cette interprétation concorde exactement avec les renseignements que l'on peut tirer de la localisation dans l'ensemble des catacombes de Rome des inscriptions de fossores: c'est bien le succès du culte des martyrs qui a assuré la fortune des fossores, et éveillé la prudence de leurs clients.

De cette fortune, les contrats gardent trace, par les indications de prix qu'ils portent à l'occasion: un sou et demi à Commodille en 426, 9, six sous à l'area de Lucine en 432 ?, 484 ou 487, 4, trois sous, sans doute en 465, pour une inscription d'origine inconnue, 95, et, dans les inscriptions non datées, quatre sous à Callixte, 34, deux sous à Cyriaque, 68 et $7^{\mathrm{b}} \mathrm{s}$ cinq cents folles, 103, et trois sous un tiers?, 108, dans des inscriptions d'origine inconnue.

Sans parler même du problème difficile de l'évaluation précise des folles ', l'interprétation de ces données est délicate, car elles sont très fragmentaires, et le plus prudent est de s'en tenir à l'ordre de grandeur des prix, qui varie de un à quatre. Je n'oserai affirmer avec G.-B. de Rossi qu'un tel éventail des prix est l'indice d'une préoccupation charitable, les plus riches surpayant leurs tombes pour dédommager les fossores des pertes qu'ils faisaient en enterrant les pauvres au-dessous du prix normal et les indigents gratuitement. Il est de même difficile d'évaluer le bénéfice tiré de leur travail par les fossores, et la tentative, d'ailleurs prudente, du même de Rossi paraît plus brillante que convaincante ${ }^{2}$. Mieux vaut sans doute reconnaître l'insuffisance de la documentation et laisser la question ouverte.

6078; IV, 11391, 12527. Les nouveaux acheteurs bénéficiaient done d'une pratique qu'ils redoutaient pour leur propre compte.

1 Le mot, on le sait, désigne à l'origine une bourse de cuir, et son équivalent en deniers n'est pas facile à fixer. On trouvera un état de la question dans I. Ruggini, Economia e società nell' Italia Annonaria", rapporti fra agricoltura e commercio dal IV al VI secolo d. C., Milan, 1961, p. 375-8: au gré des commentateurs, on obtient les équivalences les plus variées, un sou $=187$ folles, mais aussi 1 follis $=2,5,21$ ou 12500 derniers! Dans l'état actuel de la documentation, il vaut mieux donc, sans doute, renoncer à donner une équivalence.

2 G.-B. de Rossi, R.S., III, p. 552 propose d'évaluer le prix d'un bisomus à cinq à vingt jours de travail de fossor, et mentionne le graffito d'Iconius, 31, qui nous apprend qu'il lui avait fallu dix jours seulement pour aménager tout un cubiculum... aussi la marge bénéficiaire est-elle d'autant plus difficile à fixè qu'à côté de ces indications contradictoires, on ne connaît rien du prix de la matière prenière, et peu de choses de celui de la main d'œuvre (sous ('onstantin, pour de Rossi, la journée de fossor pouvait revenir à cent deniers, mais ee n'est qu'une evaluation). 
La place des fossores dans l'église de Rome.

La conversion des romains au christianisme n'avait en rien modifié leur respect des devoirs rendus aux morts: ultimum illud et maximum pietatis officium est peregrinorum et pauperorum sepultura écrit Lactance au début du IVe siècle ${ }^{1}$. Aussi le rôle des fossores peut-il être tenu pour essentiel dans la communauté chrétienne du temps, et c'est sans doute la raison qui a poussé de Rossi à se demander s'il fallait les compter au nombre des clercs. Après une étude détaillée, il concluait avec prudence qu'ils faisaient corps d'une certaine façon avec le clergé ${ }^{2}$, et son analyse a depuis été acceptée par tous. Il faut pourtant rouvrir le dossier des sources littéraires et juridiques pour apprécier exactement la portée de son jugement.

C'est au IVe siècle que l'on voit apparaître pour la première fois les fossores au milieu des autres clercs. La scène se passe en Afrique, à Cirta, lors de la perquisition effectuée le 19 mai 303 par le curator reipublicae Minutius Felix, en application du mandat qui lui avait été donné 3 , et le texte fournit les interrogatoires de l'évêque, des prêtres, des diacres et sous-diacres, et de tous les fossores dont les noms sont indiqués. Cet amalgame suffirait selon G.-B. de Rossi à prouver que les fossores sont des clercs, d'ordre mineur sans doute, et peut-être portiers, puisque leurs fonctions comprennent aussi la garde des cimetières, et que les ostiarii sont absents de l'interrogatoire. Sans doute cette interprétation est-elle un peu forcée, car, à lui seul, le hasard d'une descente de police peut vraisemblablement expliquer l'absence des uns et la présence des autres: sur le témoignage de l'interrogatoire de Cirta, on peut seulement affirmer que les fossores sont les auxiliaires habituels du clergé, ce qui ne va pas au-delà de ce que l'on peut savoir ou deviner par ailleurs.

Plus tardif, le témoignage de saint Jérôme est aussi plus précis: clerici quibus id officii erat cruentem linteo cadaver obvolviunt et fossam humum lapidibus construentes ex more tumulum parant, écrit-il dans sa première lettre ${ }^{4}$, mais est-il sûr que cette lettre, dont le récit édifiant se place à Verceil, traduit l'usage de Rome, où l'organisation de l'église,

1 Lactance, Divin. Inst., VI, 12, éd. Brandt, 1890, CSEL, XIX, p. 529.

2 G.-B. de Rossi, R.S., III, p. 535: "facevano in qualche modo corpo col clero".

3 Ie texte se trouve dans les Gesta apud Zenophilum, éd. Ziwsa, 1893, CSEL, XXVI, p. 186-187; la même scène est rapportée par saint Augustin, Contra Cresconium, III, XXIX, 33, ed. Petschenig, 1909, CSEL, III, p. 438 sq.

4 saint Jérôme, Lettre $I$, éd. Hilberg, 1910, CSEL, ILIV, p. 7. 
et celle des cimetières, pouvaient être différentes? Le renseignement ne peut donc être reçu que sous bénéfice d'inventaire.

Deux autres documents sont plus nets encore, qui insèrent les fossores dans la hiérarchie des ordres mineurs de l'Eglise. Le premier est le De VII ordinibus ecclesiae ${ }^{1}$, classé par la tradition dans les écrits de saint Jérôme, bien que ce soit une cuvre d'origine incertaine, à la date mal fixée, gauloise et du Ve siècle pour certains, espagnole et du VII siècle pour d'autres ': appliquer sa classification à l'église de Rome aux IVe et Ve siècles serait sans doute abusif. Un même jugement doit être porté sur le second document, le Chronicon Palatinum, ceuvre postérieure au moins à 574, dont l'éditeur apprécie l'antiquité notamment par le passage sur les fossores ${ }^{3}$ : nous sommes au rouet, et la prudence recommande l'abstention.

Pour finir, il reste seulement deux textes juridiques du IVe siècle $\grave{a}$ verser au dossier, mais leur signification est contradictoire. Tous deux sont de l'empereur Constance II, et traitent de la dispense de la conlatio lustralis: le premier, du 2 décembre 356, indique: clericos excipi tantum qui copiatae appellantur ${ }^{4}$, tandis que le second, du 30 juin 359 (?), porte: clerici vero, vel hi quos copiatas recens usus instituit nuncupari ita a sordidis muneribus debent immunes adque a conlatione praestari ${ }^{5}$.

En s'appuyant sur les inscriptions que j'ai données dans mon catalogue sous les nos 89 et $\mathbf{9 1}$, de Rossi a montré que ces copiatae sont les travailleurs par excellence du personnel de l'église de l'époque, c'est-àdire les fossores, et il faut sans doute retenir son identification, bien que

1 De VII ordinibus ecclesiae $=$ Ps.-Hieronymus, épist. 12, P'L, 30, col. 148, et suppl., PISS, éd. Hamman, 1960, II, 266. On relèvera particulièrement le passage: primus (...) igitur in clericis fossariorum ordo est...

2 Pour dom G. Morin, Le destinataire de l'apocryphe hiéronymien "de VII ordinibus ecclesiae", dans $R H E, 34,1938$, p. 229, l'écrit serait l'œuvre d'un pélagien adressée à Patrocle d'Arles vers 417 ; F. Griffe, L'apocryphe hiéronymien "de septem ordinibus ecclesiae", dans Bull. litt. eccl., 57, 1956, p. 213-24, nuance ces positions et pense que l'auteur de l'opuscule s'adresse à un évêque gaulois vers les années 400. Mais J. Iechner, dans Festschr. E. Eichmann, Paderborn, 1940 , p. 666-70, n. 50, penche pour une ouvre espagnole, écrite après le IIe con(ile de Séville de 619: cf. Clavis patrum latinorum ${ }^{2}, 1961$, no $^{\circ} 64$.

3 Chronicon Palatinum, éd. A. Mai, Spicil. romanum, IX, 1843, p. 133. Au passage: oportet inspicere quomodo (...) per septem gradus officii mancipandam, altario sancivierit; qui sunt ostiarius, fossarius..., l'éditeur note: locus valde notabilis ob aestimandam huius Chronicae antiquitatem.

4 Code Théodosien, XII, 1, 1 éd. Mommsen, Berlin, 1905, p. 735.

5 Ibid., XVI, 2, 15, p. 839-40. 
deux points demeurent obscurs: comment expliquer que Constance retienne l'appellation copiatae, alors qu'elle figure aussi rarement sur les inscriptions, et qu'il souligne sa nouveauté, alors qu'elle n'est attestée, semble-t-il, qu'avant la Paix de l'Eglise, soit quarante-cinq ans au moins avant son édit? ${ }^{3}$

De toute façon, le statut des copiatae demeure vague: rangés au nombre des clercs en $35 \mathbf{6}$, ils sont seulement assimilés à eux deux ans et demi plus tard. Le fait est surprenant, car on a peine à croire qu'en un aussi court laps de temps leur statut juridique ait changé, surtout dans le sens qu'indiquent les textes: il est possible en deux ans et demi de faire des fossores des clercs, mais difficilement imaginable, compte tenu des situations acquises, de les réduire à l'état laïc d'un trait de plume. Aussi a-t-on l'impression que le texte de 359 se présente sur ce point précis comme un repentir de la loi de 356 dont il reprend d'autre part les termes, et qu'il traduit une réalité, ici encore, probablement très souple et fluctuante: s'il ítablit sans conteste que le corps des fossores est assimilable à celui des clercs (et c'est à ce titre qu'il les dispense de l'impôt), il ne permet pas de connaître précisément son statut, qui devait s'adapter aux circonstances, voire aux individus. C'est une même incertitude, on le verra, qui règne sur le sort des praepositi: peut-être (faut-il ajouter sans doute?) certains fossores étaient-ils cleres, et d'autres non? Il est difficile de l'affirmer, mais il serait vain, en revanche, d'essayer de leur donner dans la hiérarchie ecclésiastique de la Rome des IVe et Ve siècles ce qu'ils n'ont vraisemblablement jamais eu: une place unique, déterminée et fixe.

Importance et déclin du rôle des fossores.

Selon les périodes, le rôle des fossores n'apparaît pas toujours en pleine lumière. Cependant, G.-B. de Rossi a su brosser à grands traits leur histoire, et, pour l'essentiel, son analyse est assurément exacte. Il est pourtant possible, en variant les éclairages, d'apporter quelques nuances et de la présenter sous un jour différent.

Ainsi, pour de Rossi, les décennies qui suivent la paix de l'Eglise marquent un tournant décisif: avec l'afflux des nouveaux convertis, si le nombre augmente, la qualité humaine baisse, chez les fossores comme

3 On trouve mention des copiate seulement sur deux inscriptions vraisemblablement antérieures à la Paix, 89, et 91, et l'on notera au contraire que l'insrription 92, pourtant entièrement rédigée en rrece, emploie le mot pódowp. 
chez les autres membres de la communauté chrétienne. Ainsi s'expliquerait l'appât du gain, qui transparaît à travers les contrats, tandis que la période précédente était celle du dévouement et du désintéressement.

N'y a-t-il pas dans cette présentation une utilisation peut-être abusive de l'argument a silentio? S'il est certain en effet que c'est seulement à partir de 338 , et plus nettement à partir du pontificat de Damase, que les contrats sont attestés, il est possible de donner de ce fait une autre explication. Je pense l'avoir déjà suffisamment exposée dans l'étude $d u$ formulaire des contrats pour qu'il soit inutile d'y revenir longuement: qu'il suffise de rappeler que c'est au moment où se développe le culte des martyrs, et souvent à proximité de leurs tombes, qu'apparaissent sur les inscriptions les mentions de vente; quand on sait le rôle joué par Damase dans le développement du culte des martyrs, même obscurs et oubliés, la coüncidence chronologique devient explication logique. Dès lors, il n'est plus nécessaire d'opposer de façon tranchée les deux périodes d'avant et d'après la paix de l'Eglise, et il est permis au contraire de souligner leur continuité.

Sans doute ne faut-il pas oublier la grande souplesse de l'organisation du travail dans les cimetières, et, partant, la grande variété des solutions qui devaient être apportées selon les cas; peut-être aussi faudrait-il faire une place à part pour les grands cimetières de la communauté, au moins aux époques les plus anciennes; sans doute enfin faut-il faire leur part et à l'évergétisme des riches et à l'œuvre d'assistance prise en charge par la communauté; il reste pourtant que, tout au long de leur histoire, les fossores ont dû vendre les produits de leur travail, loculi et cubicula, peut-être avec des bénéfices plus importants aux IVe et Ve siècles.

$\Lambda$ cette époque, ces petits artisans à l'esprit parfois individualiste apparaissent maîtres de leur cuvre et très largement indépendants: même si certains d'entre eux, peut-être, sont des cleres, leur action semble autonome par rapport au clergé. L'intervention des prêtres dans les cimetières est en effet très limitée, car l'épigraphie, au moins à ma connaissance, n'en fournit que trois exemples pour la période: à Commodille, pour le no 11, déjà mentionné, où le prêtre Maximus est témoin d'une vente, à Domitille, vers les années $360 / 70$, où une tombe est vendue sur l'ordre de deux prêtres, iussu Archelai et Dulciti presb ${ }^{1}$, et, à la même

1 ICUR, NS, III 8441. La date est fixée par la présence, à Domitille toujours, de l'épitaphe de J)ulcitius (ICUR, NS, III, 8148), dont le décès est a plarer in 369. 
époque, au cimetière de Marc et Marcellin, où une place dans un cubiculum est accordée ex iussione dmi Vincenti presbit ${ }^{1}$. Dans ces trois cas, l'intervention est de peu de conséquence: dans le contrat de Commodille, le prestige du prêtre renforce l'autorité du témoin, et à Domitille comme à Marc et Marcellin, il semble bien que l'autorité morale des prêtres ait seulement permis aux acheteurs d'obtenir, par mesure de faveur, un emplacement particulièrement disputé. Ce sont bien des exceptions qui confirment la règle, et ne touchent en fait ni aux droits ni aux intérêts des fossores, qui pendant un siècle et demi sont les seuls vendeurs de tombes dans les cimetières chrétiens.

La dernière inscription datée portant contrat signé par un fossor, 95, est peut-être de 465; la dernière épitaphe datée, 46, peut être de 513: à la fin $d u V^{e}$ et au début du VIe siècle, les fossores entrent dans l'oubli, et G.-B. de Rossi a certainement raison de rapprocher cette disparition d'un certain raidissement de la hiérarchie, émue par le commerce et le bruit fait par l'argent autour des tombes saintes; mais on expliquerait mal, me semble-t-il l'effacement des fossores et leur remplacement dans les contrats de vente par les praepositi et, accessoirement, par les prêtres, si l'on omettait de le relier également à l'effacement des cimetières chrétiens traditionnels eux-mêmes, tant il est vrai que, d'un bout à l'autre, le sort des cimetières et celui de leurs ouvriers sont liés.

Avec le tournant des années 500, en effet, commence le temps de la désaffection: les sépultures se font encore à l'occasion dans les catacombes, mais les meilleures places près des martyrs sont maintenant occupées, et la catacombe, de cimetière qu'elle était, devient surtout lieu de pélerinage. Parallèlement, le fossor est donc conduit à assumer de nouvelles tâches: sans doute avait-il toujours été le gardien du cimetière en même temps que son constructeur, comme l'indique clairement l'épitaphe n०92,

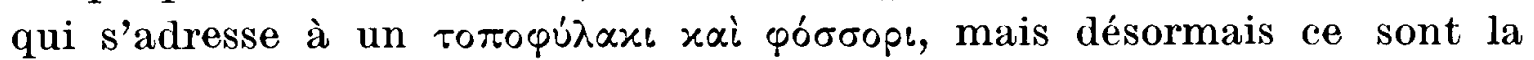
garde et l'entretien qui l'emportent sur le creusement et la sépulture. Aussi le fossor devient-il "résident", mansionarius.

Que le nouveau titre ait pu être porté par un ancien fossor, une inscription de Saint Sébastien, ICUR, NS, IV, 13412, (Fig. 5) datée de 507/8 le montre à l'évidence: ayant cessé son activité d'ouvrier des catacombes, ou ayant préféré la nouvelle appellation, jugée plus noble, Clementinus (ou sa famille) a fait rayer de son épitaphe la mention fossor pour la remplacer par masunarius = mansionarius; que le mansionarius,

$2 I C U R, N S$, IV, 11933. La date est connue cette fois aussi par l'épitaphe d'An. Vincentius à Marc et Marcellin, en 366 (ICUR, NS, IV, 11763). 
(Cliché P.C.A.S.)

Fig. 5 - Cimetière de Saint-Sébastien. Epitaphe de Clementinus, "FOSSOR" ET "MANSIONARIUS": LE MOT "FOSSOR" A ETE RATURE, ET "MASUNARIUS" AJOUTE DANS LES MARgEs.

comme le fossor auquel il succède, ait lui aussi à l'occasion vendu des tombes ressort d'une inscription d'origine inconnue, ICUR, $N S, \mathrm{I}, 1987$, l(ocus?) Faustini quem comparavit a Iulio mansionario sub conscientia prebyteri Marciani. Comme l'a bien vu de Rossi, l'expression sub conscientia est plus forte que sub praesentia employé à Commodille (mais moins forte que le iussu de Marc et Marcellin et de Domitille), et indique une certaine dépendance du mansionarius par rapport au prêtre. Il serait pour le moins aventureux pourtant de tirer une conclusion générale de cette unique inscription ${ }^{1}$.

En tout cas, la transformation du nom et celle de la fonction ne sont qu'une étape vers la disparition totale des fossores tels qu'on les a vus à l'œuvre aux IVe et Ve siècles: encore quelques décennies, et la guerre gothique et ses ruines portent aux catacombes des coups dont elles ne se relèveront plus. Sans doute les Papes multiplient-ils les efforts pour maintenir au moins le culte des martyrs, mais la répétition de ces tentatives est le gage même de leur inutilité. L'évolution économique commandait un resserrement de l'espace urbain (et, partant, des dévotions des fidèles) qui condamnait à terme le trop grand nombre de cimetières périphériques, et, avec eux, leurs ouvriers, fossores ou mansionarii, et privilégiait au contraire quelques basiliques suburbaines, bien équipées, bien

1 Il faut noter qu'en dehors de ces deux inscriptions, l'épigraphie fournit également deux épitaphes de mansionarii, ICUR, NS, II, 5177 et 5178 . 
dotées en hommes et en revenus. Avec elles, c'est dans un monde tout différent que l'on entre, un monde régi par les praepositi.

LES "PRAEPOSITI" E'T LEUR RÔLE DANS LA VENTE DES TOMBES AU VIe SIÈCLE positi ${ }^{2}$.

Catalogue topo-chronologique des interventions ou inhumations de prae-

Via Cornetia

S'aint-Pierre

1. $a^{\circ}$ 523: 'TRANSM[LNIIS], praepst basc

beati Petri (concession de tombe) ICUI, NS, II, 4184.

Via A URELIA

Saint-Pancrace

2. $a^{\circ}$ 521: FIILPPLS, praepositus beati mar. tiris Prancati (associé dans une vente à des prêtres du titre de saint (hrysogone).

ICUR, NS, II, 4279.

Via Ostinnsis

Basilique de S'aint-I'aul

3. a 498: I) IBES = Dives, praepositus

(vente diun locus) ICUR, NS, II, 4998.

4.

le même DIVES (vente)

$I C U R, N S, \quad$ I , 5167.

5.

I te même DIVES (vente: 2 sous)

ICUR, NS, II, 5168 .

6. I ce même? (nom restitué) sur une plaque de l'Area de Iucine, peutetre etrangère au cimetière.

ICUR, NS, II, 5853.

7. ao 511 ou 512: PETRlS (vente) ICUR, NS, II, 5017 .

8. a 520: Ie même PETRLS praepositus (vente: le prix a disparu) ICUR, NS, II, 5025.

9. Ie même vend un locus. ICUR, NS, II, 5171 .

10. $a^{\circ} 526$ I,ALRENTILS praepositus (épitaphe)

ICUR, NS, II, 5044.

11. a 532 S[..., praepositus, vente: 18 sous ICUR, NS, II, 5056.

1 Pour faciliter la consultation et permettre de rapides comparaisons, je suis le même ordre et les mêmes rubriques que pour les fossores.

2 A la différence du catalogue des fossores, celui-ci suit un ordre strictement chronologique, ear il n'y a pas lieu de distinguer cette fois deux périodes. 
12. $a^{\circ} 544$

13. $a^{\circ} 546 / 51$ ? $561 / 66 ? 2$

14.

15.

16.

17.

18.

19. $\left.\begin{array}{l}\text { PETRLS } 1 \\ \text { FORTLNATLS }\end{array}\right\} \begin{aligned} & \text { praepositi (ven- } \\ & \text { te d'un locus } \\ & \text { tercadens) }\end{aligned}$

Le même FORTUNATUS vend un locus 2 sous.

CIPRIANLS, praepositus (vente) DOMITIIS, diac et arcarius scae sed Apostol adque pp (ex voto) PALILS, $p b a^{3}$ (vente) Ie nême PALI US vend un locus parte verorum in aspira iovia I'repositus (vente) sur une plaque nutilée.

Prepositus anonyme (vente)
ICUR, NS, II, 5087.

$I C U R, \quad N S, \quad$ I I, 5098.

ICUR, NS, II, 5166.

ICUR, NS, II, 4788. ICUR, NS, II, 5169 .

ICUR, NS, II, 5170.

ICUR, NS, II, 5174. ICUR, NS, II, 5175 .

Extre Via Appia et Via Ardeatina

20.

Callirte, sub divo.

Vente de tombe par un $p(r a e)-$ p(ositus)

ICUR, NS, IV, 11376.

\section{Via Tiburtixa}

\section{Saint-Laurent}

21. $a^{\circ} 526$ :

STFFANIS, praepst (vente)

ICLR,

I, 1005.

22. a) 523-26:

Le même, STEFANLS, signe une réparation sur un tube de plomb,

23. daté du pontificat de Jean III. PRISCILIANUS, épitaphe.

CIL,

Diehl,
$\mathrm{XV}, 7261$. 1307.

\section{INSCRIPTIONS D'ORIGINE INCONNUE OU INCERTAINE}

24.

25.

26.

ANASTASILS praepositus (vente)
IIELPIDILS
IOHANNES praepositi

I'rositus = praepositus? (vente)
ICUR, NS, ICUR, NS, I, 420. ICUR, NS, I, 229.

1 Ce praepositus est peut-être le même que celui mentionné aux nos 7-9.

2 Cette datation proposée par l'éditeur, A. Silvagni, repose sur l'indiction $\mathrm{X}[\ldots$ qui figure sur la pierre, et sur l'identification de Fortunatus avec le praepositus du no 12.

3 L'abréviation est surprenante. Comme il n'y a pourtant aucun exemple le tombe vendue par des prêtres à Saint-Paul, je suis la leçon de l'éditeur, A. Silvagni, qui comprend praepositus. 
La société des praepositi - étude onomastique.

Le fait le plus marquant est la grande proportion de noms chrétiens, près de $60 \%$, qui se répartissent ainsi:

- nom exprimant une idée chrétienne: Anastasius, 24, déjà présent chez les fossores;

- noms tirés de la Bible: Iohannes, 25, Paulus, 16-17 (sans doute le nom est-il connu chez les païens, mais, à cette date, il est très probablement choisi pour sa signification chrétienne: I. Kaianto, On. Studies, p. 96), Petrus, 7-9 et 12, et peut-être Filippus, 2, si le nom a bien été choisi par référence à l'apôtre;

- noms de martyrs: Ciprianus, 14, Laurentius, 10, déjà attesté chez les fossores, et Stefanus, 21, 22, qui est certainement un nom chrétien au VIe siècle: I. Kaianto, On. Studies, p. 96.

On trouve peu de noms d'origine étrangère en revanche: Helpidius, 25, est le dernier représentant de l'onomastique grecque, tandis que Transmundus, 1, que Diehl, dans son commentaire (no 110) rapproche de Trasamund, trahit un nouvel apport, germanique cette fois. Les autres noms appartiennent à l'onomastique romaine courante, Dives, 3-6, Domitius, 15, Fortunatus, 13, Priscilianus, 23, et ce dernier exemple montre une fois encore le goût de la basse antiquité pour les suffixations compliquées.

A l'évidence, l'onomastique du groupe social des praepositi est aux antipodes de celle des fossores. A cela, on peut trouver deux explications qui, loin de s'exclure l'une l'autre, ont dû au contraire se renforcer mutuellement: le milieu social est différent, et les temps ont changé. Isa vogue de christianisation des noms qui touche aux IVe et Ve siècles surtout les clercs des ordres majeurs s'est étendue avec le temps jusque dans la communauté des fidèles: auxiliaires étroits du clergé ou même clercs, les praepositi du VIe siècle sont les témoins privilégiés de cette évolution.

\section{Le domaine d'activité des praepositi.}

La carte que l'on peut dresser de leurs activités (Fig. 6) contraste tout aussi nettement avec celle consacrée aux fossores. Sans doute de nouvelles découvertes pourraient-elles la modifier, mais sur des points de détail seulement, car elle traduit trop éloquemment la rétraction de la Rome du VIe siècle pour être fondamentalement inexacte. 
Il n'est qu'une catacombe, Callixte, mais sur une inscription trouvée à la surface, où l'on trouve mention d'un praepositus: les praepositi en effet sont à l'œuvre essentiellement autour des basiliques cimétériales hypéthères, non seulement à Saint-Pierre et surtout à Saint-Paul, qui

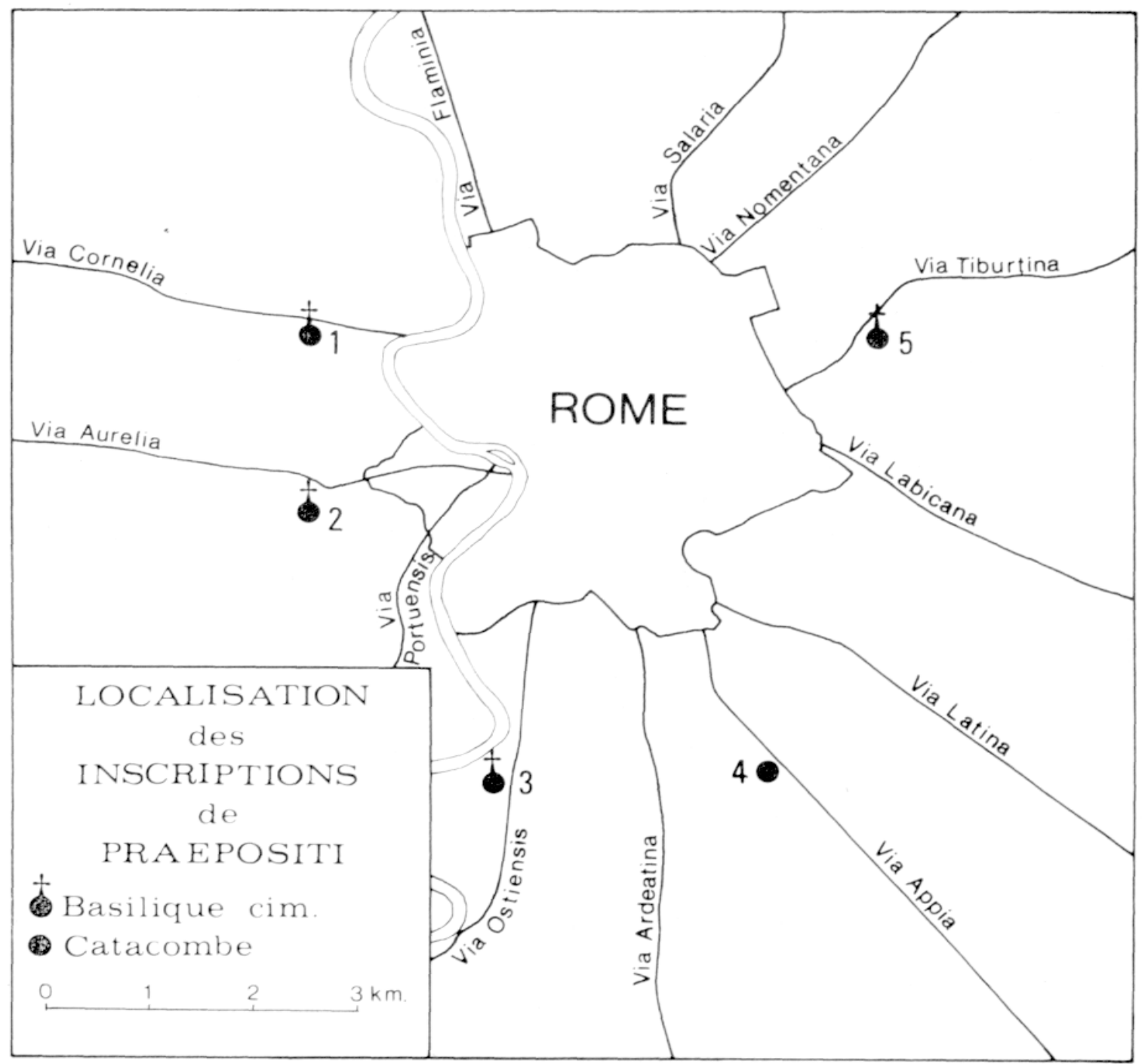

Fig. 6 - Carte de localisation des inscriptions de "PRaepositi". 1. Saint-Pierre. 2. Saint-Pancrace. 3. Saint-Paul. 4. Callixte. 5. Saint-Laurent.

étaient absentes de la carte des fossores, mais aussi à Saint-Laurent et à Saint-Pancrace. Tous ces lieux sont l'objet d'une intense activité édilitaire au début du VIe siècle, notamment sous le pontificat de Symmaque (498-514), et l'on ne manquera pas d'être frappé par la coïncidence chronologique qui existe entre l'œuvre de ce Pape et l'apparition des praepositi: au Vatican, il fait construire deux episcopia, noyaux du futur 
Palais; à Saint-Pierre, Saint-Paul et Saint-Laurent, des hospices pour les pauvres; à Saint-Pancrace enfin, une basilique ${ }^{1}$. Sans doute, dans ce dernier exemple, les éditeurs du Corpus basilicarum pensent-ils que c'était un bâtiment de peu d'importance ${ }^{2}$, mais la coïncidence presque exacte de sa construction avec la mention d'un praepositus beati martiris Prancati fait penser que la création de la fonction est liće, au moins dans ce cas, à celle de l'édifice.

Cette localisation très particulière des praepositi confirme l'évolution historique que l'étude des fossores a permis de discerner: au moment où les catacombes perdent graduellement leur office principal de cimetière, la relève est assurée essentiellement par le succès des basiliques suburbaines, et, tandis que dans les anciennes nécropoles les fossores assurent essentiellement un rôle de gardiennage, les praepositi, dans les nouvelles, vendent des tombes, creusées et équipées par d'autres.

\section{L'organisation du travail des praepositi.}

Le trait le plus marquant est que leur fonction, en effet, est fondamentalement différente de celle des fossores: les peaepositi ne sont pas de petits artisans indépendants, mais des préposés à l'entretien des basiliques, et, accessoirement, à l'inhumation dans leur enceinte: à ce titre, ils se trouvent sans doute à la tête d'un petit personnel presque inconnu: seul un mansionarius, inhumé à Saint-Paul, ICUR, NS, II, 5177, mansion $a p($ osto $) l i$ basilicae, pourrait en avoir fait partie. Il est vraisemblable que ce mansionarius avait à Saint-Paul une position subordonnce aux praepositi, à la différence de ses collègues, gardiens des catacombes, qui devaient jouir d'une certaine indépendance.

Ies autres fonctions des praepositi ont cité suffisamment étudiées par G.-B. de Rossi pour qu'il soit inutile d'y revenir ${ }^{3}$. Qu'il suffise de rappeler qu'ici encore une même charge recouvre des réalités très différentes. Au sort des praepositi des grandes basiliques (auxquels il faut joindre sans doute celui de Callixte) s'oppose en effet celui du praepositus

1 Liber I'ontificalis, éd. I. Iuchesne, I, p. 262: item episcopia in eodem. loco (basilica s. Petri) dertra levaque fecit (...) eodem tempore fecil basilicam Sancti P'ancrati; p. 263: item ad beatum I'etrum et beatum I'aulum et ad Sanctum Iaurentium pauperibus habitacula construxit.

2 R. Krautheimer, S. Corbett, W. Frankl, Corpus basilicarum christianarum Rome, III, Rome-New York, 1967, p. 172: "it would seem to have been a small structure".

3 G.-B. de Rossi, R.S., III, p. 520 sq. 
de Saint-Pancrace: les premiers semblent indépendants du clergé romain, quand le dernier, selon le mot de Rossi, reste sous l'“ingerenza o sorveglianza " des prêtres du titre de Saint-Chrysogone ${ }^{2}$. Dans la vente où il apparaît, il intervient en effet en cinquième position, après tous les prêtres rangés par ordre hiérarchique; en outre, on le verra, les autres ventes effectuées à Saint-Pancrace au début du VIe siècle sont le fait des prêtres; inversement, à Saint-Pierre, à Saint-Paul et à Saint-Laurent, les prêtres n'apparaissent jamais; à Callixte, rarement, sinon jamais ${ }^{2}$. Ies autres praepositi dépendent peut-être directement du Pape, fondateur ou protecteur des édifices dans lesquels ils opèrent: la chose est certaine pour celui de Saint-Pierre, 1, nommé après le Pape sur l'inscription, parce qu'il agit sur son ordre, très vraisemblable au moins pour ceux de Saint-Paul, dont l'un, 15, était arcarius du Saint Siège, probable pour Callixte et même pour Saint-Laurent.

On notera d'autre part la souplesse de l'organisation: le plus souvent, un praepositus suffit à la tâche, mais, quand elle est trop lourde, on n'hésite pas à doubler les postes. C'est le cas pour Helpidius et Iohannes, 25, sur une inscription d'origine inconnue; c'est le cas surtout pour la basilique de Saint-Paul, où en 544, 12, on trouve deux titulaires, Petrus et Fortunatus. Ici, l'interprétation du document est difficile: faut-il penser qu'il y avait habituellement deux praepositi à Saint-Paul pour se partager la tâche et que, à l'ordinaire, seul figurait sur les actes de vente le nom de celui qui avait présidé à la tractation? ou bien, si le Petrus de cette inscription est le même que celui attesté en $511 / 12$, 7 , et en 520, 8, faut-il penser que Fortunatus est une sorte de coadjuteur qui lui a été donné après trente-trois ans au moins de services dans la basilique?

Les contrats de vente passés par les praepositi.

On retrouve très précisément les types de contrats examinés à propos des fossores, tant il est vrai que la tradition et le conservatisme sont de règle à la fois dans la rédaction des textes funćraires et dans celle des textes juridiques.

Cependant, l'importance relative de chaque catégorie a profondement changé: ainsi, les contrats du type $N$. comparavit (ou emit) locum ne sont plus représentés que par trois exemplaires, 7 (a' 511/12), 8,

1 Ibid., p. 522.

2 Un seul exemple à Callixte, et douteux, sur une inscription mutilée, ICIR, NS, IV, 11502. 
( $a^{\circ} 520$ ) et 26, tandis que l'emportent, et de très loin, les formules hunc locum... sibi comparaverunt, 2, ( $\left.\mathrm{a}^{\circ} 521\right), 11$ ( $\mathrm{a}^{\circ}$ 532) ou leurs variantes, locus $N$. quem se vivus comparavit 3 (a 498), 12 (a 544), 13 (a 546/51 ou 561/66?), 4, 16?, 18?, locus $N$. comparaverunt, 6, locus $N$. quem emit, 25, ou, avec l'emploi du participe passé, [locum] comparatum, 9, 19, et emptu locu, 17. Enfin, on notera une formule rare, réservée à une intervention conjointe du Pape et d'un praepositus, 1, concessum locum.

Le succès des nouvelles formules et la quasi-disparition des anciennes confirment la chronologie relative proposée pour les inscriptions de fossores, même si l'on peut expliquer aussi le succès du terme locus par les conditions nouvelles d'inhumation: plus encore que le loculus de catacombe, le dalle banale et interchangeable du cimitière ou de la basilique sub divo mérite cette appellation vague.

En revanche, on ne trouve plus guère trace des clauses que les acheteurs, par défiance, avaient introduites dans les contrats passés avec les fossores: il n'y a de témoins que pour la vente de Saint Pancrace, 2, qui paraît avoir revêtu un cractère particulièrement solennel, si l'on en juge par le nombre des assistants; d'autre part, il n'y a pas d'indication précise de lieu, sauf pour le no 17 , où l'on doit lire sans doute in absida; enfin, les indications de prix sont rares: deux sous vers 546/66 à Saint-Paul, 13, et dans la même basilique à des dates indéterminées, deux sous, 5, dix-huit sous, 11, trois sous un tiers, deux sous un tiers, trois sous (ICUR, NS, II, 5001, 5173, 5172, sur des plaques où le nom du vendeur a disparu). L'ordre de grandeur reste le même que pour les ventes des fossores, à l'exception du no 11 au prix anormalement élevé.

Sans doute faut-il penser à la lumière de ces indications que la nouvelle organisation inspirait plus de confiance que celle de la période précédente, encore que la concurrence autour des tombes restât sans doute toujours vive, comme on peut le voir peut-être à un léger indice: l'autre face de la dalle qui sert d'épitaphe à Laurentius, 10, porte la mention [l]ocus Laurent[i], et on peut penser avec l'éditeur que cette inscription avait été gravée dans l'attente du jour de la depositio (et de la rédaction sur l'autre face de l'épitaphe proprement dite), pour soustraire l'emplacement à la convoitise d'autres candiclats.

La place des praepositi dans l'église de Rome.

S'il est clair que l'un des praepositi de Saint-Paul, Domitius, 15, était clerc, et plus précisément diacre, diac et arcarius scae sed Apostol, le statut des autres est inconnu. Aucun texte canonique, liturgique ou juridique ne fournit de renseignements précis, et on peut seulement remar- 
quer avec de Rossi que leur fonction, en tout cas, n'mpliquait pas nécessairement le sacerdoce.

On peut donc imaginer pour eux une solution du type de celle envisagée pour les fossores: l'appartenance au sacerdoce devait être affaire de circonstances, voire d'individus. Peut-être les praepositi des basiliques les plus importantes étaient-ils clercs? Ce n'est là qu'une hypothèse, et il serait vain, sans doute d'imaginer une règle précise.

Importance et déclin du rôle des praepositi.

Les prapositi n'apparaissent dans la documentation qu'au VIe siècle: attestés pour la première fois en 492, ils semblent s'évanouir dès les années 561/566. Il est possible de formuler une hypothèse au moins pour expliquer leur brève carrière ${ }^{1}$.

Les praepositi en effet sont essentiellement les hommes des basiliques sub divo que les Papes construisent, restaurent ou reconstruisent avec obstination au $\mathrm{VI}^{\mathrm{e}}$ siècle, pour essayer de maintenir autour des cimetières extra muros non seulement des centres de culte (et partant des sépultures), mais aussi des faubourgs assez peuplés pour garder à la Ville son équilibre passé. Mais l'excès des ruines dues aux guerres comme les changements économiques qu'elles entraînent sont plus forts que la volonté des hommes, et l'effort pontifical se solde par un échec: dès le VII siècle, les faubourgs se dépeuplent, et les inhumations commencent à l'intérieur de la Ville. L'échec de la tentative obstinée, mais inutile, de la Papauté peut permettre d'expliquer la disparition des praepositi qui en furent les exécutants.

\footnotetext{
L'“ ingerence et La surveillance" des prettres et du Pape DANS LES VENTES DE TOMBES (IVe-VIIe SIÈCLES) ${ }^{2}$.

Catalogue topo-chronologique des interventions des prêtres et du Pape dans les cimetières.

$$
\text { A. - Interventions des prêtres }
$$
}

1 On la recevra avec prudence, car les inscriptions datées actuellement connues sont peu nombreuses, et quelques trouvailles suffiraient éventuellement à bouleverser la chronologie.

2 Je me borne à étudier le rôle, d'ailleurs limité, des prêtres (et à l'occasion du Pape) dans la vente des tombes. Aussi ne trouvera-t-on ici aucune étude de leur groupe social, mais un catalogue sommaire de leurs interventions, et les commentaires qu'il demande. 


\section{Via Aurelia}

Saint-Pancrace

1. a 521 :

$\begin{array}{ll}\text { PETRLS } & \text { prêtres du titre } \\ \text { CRISOGONLS } & \text { des. Chrysogo- } \\ \text { CATELILS } & \text { ne (vente aux } \\ \text { GALDIOSLs } & \begin{array}{l}\text { cotés diun prae- } \\ \text { positus) }\end{array}\end{array}$

2. $a^{0} 522$ :

Le mêne PETRLS (vente)

3. Vers 520:
Ie même PETRLs et MICINL's

(autorisation (concessum) de vente)
ICUR, NS, II, 4279.

ICUR, NS, II, 4280.

ICLR, NS, II, 4312.

Via Portuexsis

Pontien

4.

Presbyter, dont le nom est perdu (vente)

ICUR, NS, II, 4674.

Via Ostiensis

Commodille

5. IVe.Ve sièc.? MAXIML's (témoin à une vente)

6. $a^{\circ} 527$ Prêtre intervenant dans une venou 528: te 1 .

ICUR, NS II, ,6096.

ICUR, NS, II, 6088.

Via Ardentisa

Domitille

7. vers 360 2: AR(HEIALS, autorisent (iusDILCITIES $\quad(s u)$ une vente. ICUR, NS, III, 8441.

Extre Via nppia et Via Ardeatina

Callixte

8.

Vente d'une tombe par un prêtre? (titulus très mutilé) ${ }^{3}$.

ICUR, NS, IV, 11502.

Ware ol Warcellin

9. vers 360 ${ }^{4}$ : VINCENTILS autorise (ex ius. ICUR, NS, IV, 11933. sione) une vente

2 I'inseription est mutilée et restituce ainsi par A. Silvagni: locus comparalus a P]etro primic tit Scae [Sabinae? concessus su]b pro P'aulo. Le prêtre semble donc avoir seulement autorisé et supervisé la vente.

2 Pour l'établissement de cette date, voir supra, p. 578, n. 1.

3 Les restitutions sont considérables: locus Crescen]tionis $q[u e m$ emit a... presb] T' sce Ba[lbinae: aussi peut-être faut-il rejeter cette incription.

4 Pour l'établissement de cette date, voir supra, p. 578, n. 2. 
10.

$$
\ldots \text { JORIS (vente) ICUR, NS, IV, } 11845 .
$$

Via A PPIA

Saint-Sébastien

11.

HIIAA RLS, prêtre? (selon les resti- ICUR, NS, V, 13555. tutions de l'éditeur) (vento)

Prétextat

12.

INNOCENTILS autorise (iubente) ICUR, NS, V, 14568. les ventes d'un bisomus et d'un monosomus

Via I,aBicana

Pierre et Marcellin

13.

Prêtre dont le nom est perdu Diehl, 3768 adn. (autorisation de vente) (?)

Via Tiburtina

Cimetière d'Hippolyte

14. a 489: Prêtre du titre de Sainte-Praxède Diehl, $3766 \mathrm{~A}$. (vente)

EGIIse intra muros (?) 1

Sainte-Cécile au Trastevere

15. ao 619: VICTOR, archiprêtre du titre de Rossi, R.S. III, p. 552. de Sainte-Cécile (vente: 6 sous)

16. EXIIAARATLS (vente: 4 sous 1/3) IOUR, NS, I, 131.

INSCRIPTIONS D'ORIGINE INCONNUE

17.

Vente faite par un mansionarius

ICUR, NS, I, 1987. sous la responsabilité (sub con.

18. scientia) du prêtre Marcianus PETRUS, archiprêtre (vente) ICUR, NS, I, 1014.

1 Pour G.-B. de Rossi, R.S., III, p. 552, l'inscription no 15, conservée aujourd'hui à Sainte-Cécile au 'Trastevere, peut-être originaire de l'église même, car les inhumations intra muros avaient déjà commencé en 619. Peut-être en cst-il de même pour le no 16 ? 


$$
\text { B. - Principales interventions du Pape }
$$

Via Cornelia

Saint-Pierre

19. a 523: Concession d'une tombe par l'in- ICUR, NS, II, 4184. termédiaire du praepositus

Transm[undus]

20. a $^{\circ} 563$ :

Concession d'un locus

ICUR, NS, II, 4186.

21. Concession d'une tombe (le nom ICUR, NS, II, 4236. du Pape est perdu)

Via Aureitia

Saint-Pancrace

22. a 537: Concession d'une tombe

ICUR, NS, II, 4283.

Extre Via Appia et Via Ardeatina

Callixte

23. avant 304: Concession (iussu) d'un cubiculum ICUR, NS, IV, 10183. au diacre Severus

INSCRIPTION D'ORIGINE INCONNUE OU INCERTAINE

24. a" 522: Donation d'une tombe ICUR, NS, I, 749.

Modalités des interventions.

Pour reprendre le mot de de Rossi, déjà cité, ingérence et surveillance sont bien les deux attitudes adoptées par les prêtres ou le Pape par rapport au commerce des tombes. Pourtant, il y a plusieurs degrés dans les interventions, qui vont de la simple présence à une vente comme témoin, à la vente de tombe pure et simple.

La surveillance des prêtres s'exerce à travers le rôle de témoin assumé par Maximus à Commodille, 5, (Fig. 7) ou par l'autorité morale d'un Marcianus, 17, qui est intervenu dans la vente faite par un mansionarius ${ }^{1}$. Plus proches de l'ingérence déjà sont les interventions où le prêtre a ordonné une vente: c'est le cas des inscriptions portant le formulaire iussu, 23 (avant 304, pour une intervention du Pape), 7 (vers 360/70),

1 Sur l'étendue de cette autorité morale, voir supra, p. 579, mon commentaire de l'expression sub conscientia presbyteri employée dans l'inscription. 


\section{Illustration non autorisée à la diffusion}

(Cliché P.C.A.S.)

Fig. 7 - Crmetière de Commodille. Epitapile rédigée sous Forme de contrat de vente: Caianus a achete, pour lui et pour sa femme, UNE TOMBE $i$ DEUX PIACES AU "Fossor" Adeodatus, EN PREsence I) PRÊTRE MAXimus.

ex iussione, 9 (vers 360/70) ou iubente, 12. Encore que l'expression soit assez vague, il faut sans doute comprendre que le produit de la vente a bien été encaissé par l'artisan qui a effectué les travaux, et que le prêtre a seulement usé de son autorité pour faire accorder à son protégé une sépulture dans un endroit disputé.

Plus tard, un pas supplémentaire est franchi par les concessions accordées par le Pape ou par des prêtres. On les trouve dans les formules du type: locum concessum, 3 (autour de 520), et, pour les interventions du Pape, 19 (a 523), 20 ( (a ${ }^{\circ}$ 563), concedente, 22 (a 537 : concession du Pape), et peut-être concessum sub presbytero N., 6 (a 527/8), 13: mais ces deux derniers exemples ne sont que des restitutions, d'ailleurs vraisemblables. Au même type d'intervention apparaît la donation pontificale (donavit) no 24 (a 522 ).

Faut-il penser que des formulaires de ce type trahissent l'intervention charitable du clergé et que ces concessions sont gratuites? ou, au contraire, que la concession suppose de la part de celui qui en bénéficie un don qui n'est pas mentionné? Il est difficile de trancher: à Saint Pan- 
crace, la concession no 3 est accordée par le même prêtre, Petrus, qui a assuré les ventes no 1 et 2 ; on serait tenté d'en déduire que le terme de concession recouvre la même contrepartie financière qu'une vente, mais il est également possible de supposer que, dans ce cas précis, Petrus a pris une mesure de faveur pour des fidèles qui se trouvent etre aussi des confrères, les prêtres Bonifatius, Balentinus et Xystus.

Enfin, dans un cas sur trois environ, les prêtres interviennent directement dans les ventes des tombes, qui sont indiquées par les formules comparavit/erunt, 4, 14, 15, 16 ou se vivus/i comparavit/erunt, 1 (a 521), 2 (a 522$), 10,11$ ?, et dont le prix est deux fois précisé: quatre sous un tiers, 16, et six sous, 15, à Sainte Cécile au Trastevere. Il ressort clairement de cette proportion, et plus clairement encore du rapprochement avec les quelques cent dix inscriptions conservant des noms de vendeurs, que la vente des tombes est pour les prêtres une activité occasionnelle (sant doute liée à des circonstances exceptionnelles que les documents ne permettent pas de connaître) et que leur ingérence directe dans les affaires de vente dans les cimetières est en fin de compte très limitée.

Localisation des interventions (Fig. 8).

Si la localisation des interventions du Pape, non seulement à SaintPierre et à Callixte, mais aussi à Saint-Pancrace, à une date où les nouvelles fondations pontificales venaient d'y ctre effectuces, ne cause guère de surprises, celles des prêtres, très dispersées malgré leur petit nombre, ne répondent visiblement à aucune intention cohérente, et trahissent par leur éparpillement le rôle du hasard et de l'occasion: ainsi est confirmé le caractère exceptionnel de leur actions dans les cimetières.

Deux séries d'interventions pourtant échappent à ce jugement: ce sont les nos 1-3 à Saint-Pancrace, et 15-16 à Sainte-Cécile au Trastevere, car, dans ces cas, la vente des tombes semble avoir été non un accident, mais une activité habituelle des prêtres. A Saint-Pancrace, je l'ai dit, l'autorité du praepositus semble bien inférieure à celle de ses collègues des autres basiliques: associé, mais subordonné, aux prêtres pour la première vente, il disparaît dans les suivantes, et les prêtres du titre de Saint-Chrysogone qui, pour des raisons inconnues, préfèrent agir à sá place manifestent une autorité unique à cette date. $A$ u début du VII siècle, en revanche, les prêtres qui vendent une tombe à Sainte-Cécile au Trastevere même, si l'on admet l'hypothèse de de Rossi ${ }^{1}$ (comme

1 Sur la nature do cettr hypothese, voir supra, p. 589, n. 3. 


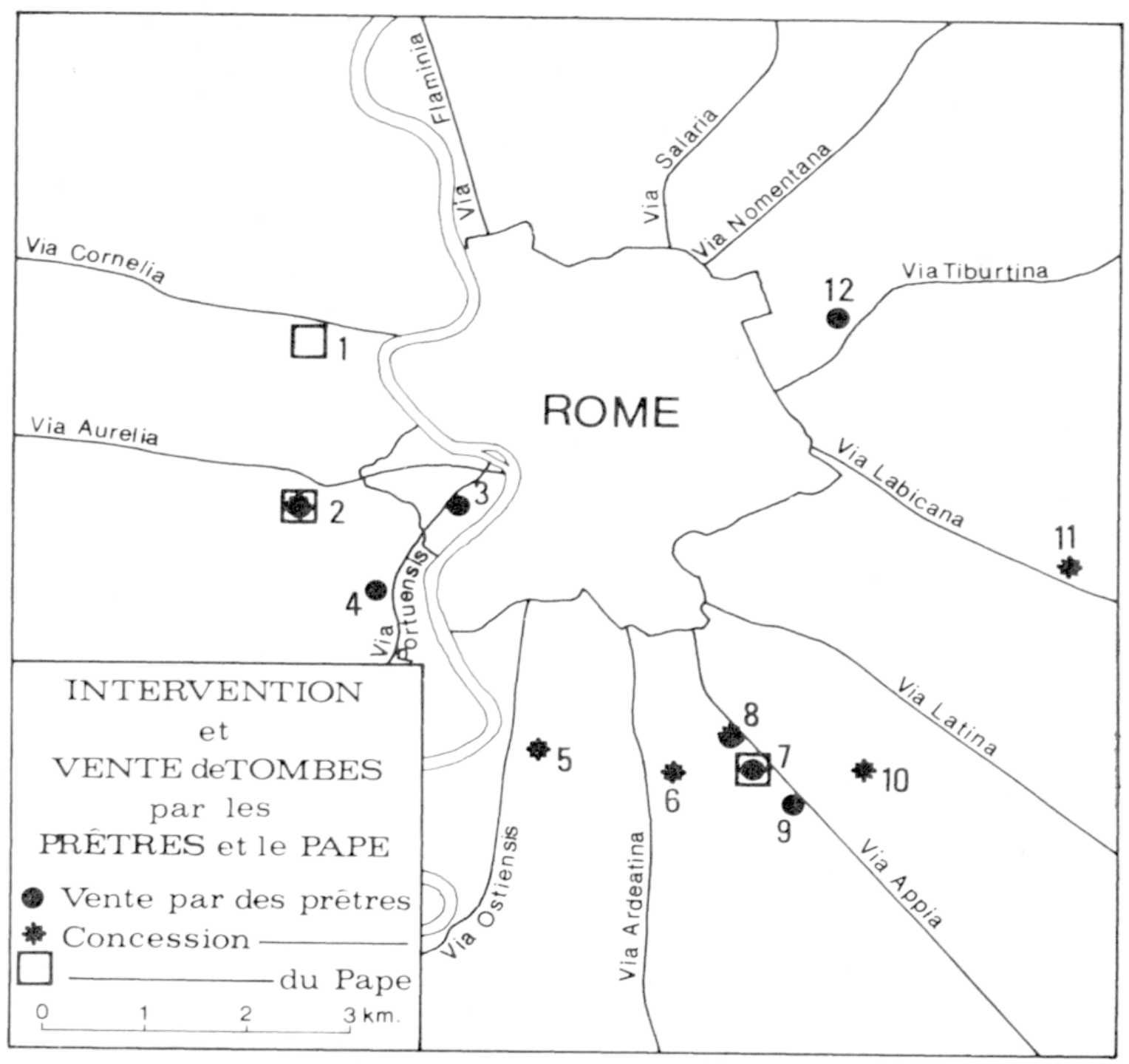

Fig. 8 - Carte de localisation des interventions et ventes de tombes faites par les prêtres ou le Pape.

1. Saint-Pierre. 2. Saint-Pancrace. 3. Sainte-Cécile au Trastevere. 4. Pontien.

5. Commodille. 6. Domitille. 7. Callixte. 8. Marc et Marcellin. 9. Saint-Sébastien. 10. Prétextat. 11. Pierre et Marcellin. 12. Hippolyte.

peut-être aussi leurs collègues de la vente no 16 , qui peut dater de la fin du VIe siècle) sont au contraire les témoins d'un usage récent. Dans les églises urbaines où ont lieu désormais les inhumations, ce sont en effet les prêtres qui en ont la charge, comme les praepositi l'avaient pour les basiliques suburbaines. Au terme chronologique de cette enquête, c'est donc à nouveau un usage tout différent qui apparaît, et il n'est pas inutile MEFRA 1974, 1. 
sans doute pour finir de résumer les grandes lignes de l'́rvolution qui l'a créé.

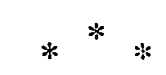

Tout au long des quatre siècles considérés dans la présente étude, le paysage de Rome a bien changé. La splendeur de la capitale impériale a péri avec les invasions, tandis que ses habitants se sont réfugiés dans des quartiers resserrés et plus aisément défendables que la muraille aurélienne, devenue trop large pour le corps amaigri de la citć.

Jadis, la ville des morts entourait la ceinture de jardins qui enserrait le centre urbain et politique de la cité, et c'est dans cette auréole périphérique que les chrétiens avaient creusé le tuf pour implanter leurs catacombes. Quand vinrent les guerres et leurs dévastations, les nécropoles furent frappées au même titre que la Ville: et ecclesias et corpora martyrum sanctorum exterminatae sunt, dit un passage fameux du Liber Pontificalis ${ }^{1}$. Nullement découragée par les ruines, la Papauté a cru un moment pouvoir au moins sauver quelques secteurs privilégiés, et a multiplié les efforts aussi bien pour maintenir le souvenir des martyrs que pour fixer ou retenir les vivants aux avant-postes de la cite. Mais les modifications économiques et politiques furent les plus fortes, et la tendance à l'abandon l'emporta: malgré quelques tentatives désespérées de restauration des cimetières dans le courant du VIIe siècle, dès le début de ce siècle, les romains rentrent dans l'enceinte trop vaste, et les morts à leur tour prennent place dans la Ville. La levée de l'interdit religieux sur la sépulture intra muros, vieux d'au moins un millénaire (il est attesté dès la I tion historique.

D'une évolution aussi radicale, l'administration de la vente des tombes dans les cimetières chrétiens garde elle aussi le trace. Tout au long de cette longue période, elle a connu de profonds changements qui sont bien connus, au moins dans leurs grandes lignes: on voit clairement la situation de départ comme celle de l'arrivée et les principales étapes des transformations. Rien ne serait plus faux cependant que d'imaginer une évolution linéaire, due à une volonté constante et à une politique arrêtée; nul doute au contraire qu'elle s'est accomplie vaec la plus grande souplesse,

1 Liber Pontificalis, éd. I. I)uchesne, I, p. 291. 
parfois sans doute avec des accélérations, ou des retours en arrière, d'autres fois en respectant des situations acquises, de sorte qu'aux périodes de transition plusieurs types d'exploitation coexistaient probablement.

En un mot, comme la réforme de l'administration de Rome, qui a assuré le passage des régions augustéennes aux régions ecclésiastiques, la réforme de l'administration des ventes dans les cimetières a manifesté le plus grand pragmatisme: le souci d'épouser des réalités changeantes a été prédominant, de sorte qu'à chacune des étapes de l'évolution des cimetières, leur administration présente un visage différent.

Aux IVe et Ve siècles, au moment où l'église entre dans la paix, et bénéficie de l'appui du pouvoir politique, les catacombes poussent leurs ramifications, équipent leurs cubicula, et creusent leurs retro-sanctos au long de toutes les voies consulaires, où l'on peut voir à l'œuvre des équipes entières d'ouvriers: c'est la période de plein épanouissement des cimetières suburbains, qui deviennent avec le temps à la fois nécropoles et lieux de culte et de pélerinage auprès des corps saints. A cette date, la vente des tombes est l'affaire quasi-exclusive des fossores: soumis de loin à l'autorité morale des prêtres, et beaucoup plus rarement à leur surveillance effective, ces petits artisans indépendants d'origine populaire, laïcs pour la plupart, vivent de la vente des tombes qu'ils ont aménagées.

Avec le sixième siècle, tandis que les inhumations deviennent plus rares dans les catacombes, les nécropoles les plus actives sont situées à l'intérieur et autour des basiliques suburbaines entretenues ou fondées par les Papes. A la faveur de cette mutation, l'administration des ventes dans les cimetières change aussi: tandis que les fossores deviennent de plus en plus de simples gardiens chargés de l'entretien des lieux vénérés, et que certains d'entre eux au moins prennent le titre de "résidents" (mansionarii), dans les basiliques suburbaines, l'administration est habituellement confiée, sous la surveillance des prêtres ou du Pape, à des praepositi, cleres ou laïcs, à qui la vente des tombes est également dévolue: aussi se trouvent-ils à la tête d'un petit personnel d'artisans subordonnés, dont certains portent, par analogie avec la nouvelle appellation des fossores, le titre de mansionarii.

Dès le début du VII'e siècle enfin, quand s'affirme le repli sur Rome et que commencent les inhumations dans les églises urbaines, ce sont les prêtres chargés de l'administration de ces églises qui assurent normalement la vente des tombes, dans des conditions analogues à celles des praepositi pour la période précédente. 
Ainsi, le parallélisme est parfait entre les bouleversements de la topographie urbaine et ceux qui touchent l'administration de la vente des tombes dans les cimetières: dans les deux cas, c'est un même mouvement de concentration qui est perceptible. Tandis que l'espace urbain se rétracte et que la Ville des Césars devient la Rome du Moyen-Age, les prêtres, qui n'avaient au début qu'un rôle de surveillance sur la vente des tombes, interviennent de plus en plus nettement, et, pour finir, prennent en main cette cuvre d'administration. Ici aussi, au terme de l'évolution que j'ai essayé de retracer, l'usage médiéval naît des pratiques antiques. 